\title{
MMSE-based Transceiver Design Algorithms for Interference MIMO Relay Systems
}

\author{
Khoa Xuan Nguyen, Student Member, IEEE, Yue Rong, Senior Member, IEEE, and Sven Nordholm, Senior \\ Member, IEEE
}

\begin{abstract}
In this paper, we investigate the transceiver design for amplify-and-forward interference multiple-input multipleoutput (MIMO) relay communication systems, where multiple transmitter-receiver pairs communicate simultaneously with the aid of a relay node. The aim is to minimize the mean-squared error (MSE) of the signal waveform estimation at the receivers subjecting to transmission power constraints at the transmitters and the relay node. As the transceiver optimization problem is nonconvex with matrix variables, the globally optimal solution is intractable to obtain. To overcome the challenge, we propose an iterative transceiver design algorithm where the transmitter, relay, and receiver matrices are optimized iteratively by exploiting the optimal structure of the relay precoding matrix. To reduce the computational complexity of optimizing the relay precoding matrix, we propose a simplified relay matrix design through modifying the transmission power constraint at the relay node. The modified relay optimization problem has a closedform solution. Simulation results demonstrate that the proposed algorithms perform better than the existing techniques in terms of both MSE and bit-error-rate.
\end{abstract}

Index Terms-Interference channel, MIMO relay, MSE

\section{INTRODUCTION}

Multiple-input multiple-output (MIMO) systems can provide a significant improvement in the system data rate even under conditions of interference [1]. By incorporating relay nodes in a MIMO system, the network coverage and reliability can be significantly improved [2], [3]. In a MIMO relay system, communication between source nodes and destination nodes can be assisted by single or multiple relays equipped with multiple antennas. The relay nodes can either decodeand-forward (DF) or amplify-and-forward (AF) the relayed signals [4]. In the AF scheme, the signals received at the relay nodes are simply amplified (including a possible linear transformation) through the relay precoding matrices before being forwarded to the destination nodes. Therefore, in general the AF strategy has lower complexity and shorter processing delay than the DF strategy.

For single-user two-hop MIMO communication systems with a single relay node, the optimal source and relay precoding matrices have been developed in [5], [6]. For a singleuser two-hop MIMO relay system with multiple parallel relay nodes, the design of relay precoding matrices has been studied in [7], [8]. Recent progress on the optimization of AF MIMO relay systems has been summarized in [3].

The authors are with the Dept. of Electrical and Computer Engineering, Curtin University of Technology, Bentley, WA 6102, Australia, e-mails: khoa.x.nguyen@ieee.org, y.rong, s.nordholm@curtin.edu.au.

This work was supported under the Australian Research Council's Discovery Projects funding scheme (project numbers DP110100736, DP140102131).
For MIMO interference channels, the idea of interference alignment (IA) [9] was developed for interference suppression by arranging the desired signal and interference into corresponding signal spaces. The idea of IA has been applied in interference MIMO relay systems in [10], [11]. However, there is still no general solution for IA as a number of conditions must be met. One main reason is that the number of dimensions required for IA is very large and it depends on the number of independent fading channels. This leads to high computational complexity and infeasibility in practical systems. In [12], an iterative algorithm has been proposed to optimize the source beamforming vector and the relay precoding matrices to minimize the total source and relay transmit power such that a minimum signal-to-interference-plus-noise ratio (SINR) threshold is maintained at each receiver. An iterative transceiver design algorithm has been developed in [13] to minimize the total interference in interference MIMO relay systems. Recently, a robust joint transceiver design has been proposed in [14] for interference MIMO relay networks in the presence of statistical channel state information (CSI) errors.

In this paper, we consider an interference MIMO relay communication system where multiple transmitter-receiver pairs communicate simultaneously with the aid of a relay node. The transmitters, receivers, and the relay node are equipped with multiple antennas. Based on the fact that the raw bit-error-rate (BER) is closely related to the mean-squared error (MSE) of the signal waveform estimation at the receivers, the minimal MSE (MMSE) is chosen as the design criterion. We aim at optimizing the transmitter, relay, and receiver matrices to suppress the interference and minimize the sum MSE (SMSE) of the signal waveform estimation at the receivers, subjecting to transmission power constraints at transmitters and the relay node.

Since the transceiver optimization problem is nonconvex with matrix variables, a globally optimal solution is computationally intractable to obtain. To overcome the challenge, we propose an iterative transceiver design algorithm through solving convex subproblems. In each iteration of this algorithm, we first update the receiver matrices based on the transmitter and relay matrices from the previous iteration. Then we optimize the relay matrix based on its optimal structure, the transmitter matrices from the previous iteration, and the receiver matrices in this iteration. Finally, the transmitter matrices are updated. The MSE and bit-error-rate (BER) simulation results demonstrate that the proposed algorithm converges in a few iterations. Note that although an iterative algorithm has been 
proposed in [15] for interference MIMO relay systems, the optimal structure of the relay matrix is not exploited in [15].

To reduce the computational complexity of optimizing the relay precoding matrix, we propose a simplified relay matrix design through modifying the transmission power constraint at the relay node. The modified relay optimization problem is suboptimal, but it is convex and has a closed-form solution. Simulation results show that the simplified relay matrix design has a slightly worse performance than the optimal relay matrix in terms of the system MSE and BER. However, the computational complexity of the simplified algorithm is much smaller than that of the optimal relay design for interference MIMO relay systems with a large number of transmitter-receiver pairs. To the best of our knowledge, the proposed simplified relay matrix design cannot be found in existing works on transceiver optimization for interference MIMO relay systems such as [12]-[15].

The rest of this paper is organized as follow. The system model and problem formulation are introduced in Section II. The proposed joint transmitter, relay, and receiver matrices design algorithms are presented in Section III. In Section IV, we discuss the possibility of extending the proposed algorithms to two general scenarios: (a) systems with imperfect CSI; (b) systems with multiple relay nodes. Simulation results are presented in Section V to demonstrate the performance of the proposed algorithms. Conclusions are drawn in Section VI. Throughout this paper, scalars are denoted with lower or upper case normal letters, vectors are denoted with bold-faced lower case letters, and matrices are denoted with bold-faced upper case letters. Superscripts $(\cdot)^{T},(\cdot)^{H}$, and $(\cdot)^{-1}$ denote matrix transpose, conjugate transpose, and inverse, respectively, $\operatorname{tr}()$ stands for matrix trace, vec() stacks columns of a matrix on top of each other into a single vector, $b d()$ denotes a blockdiagonal matrix, $\otimes$ represents the Kronecker product, $E[$ ] denotes the statistical expectation, and $\mathbf{I}_{n}$ stands for the $n \times n$ identity matrix.

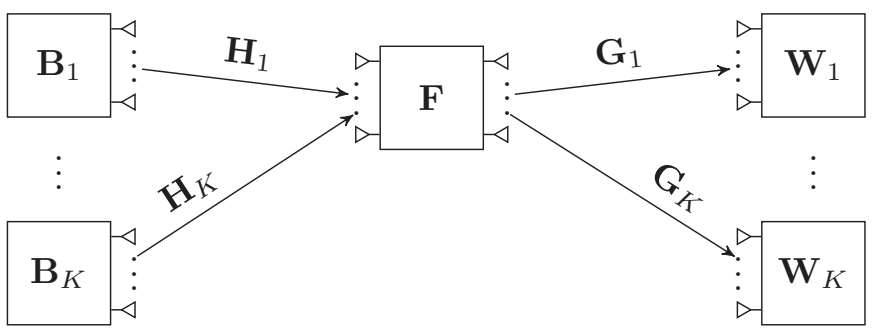

Fig. 1. Block diagram of an interference MIMO relay system.

\section{SyStem MOdEL AND PROBlem Formulation}

We consider a two-hop interference MIMO relay communication system where $K$ transmitter-receiver pairs communicate simultaneously with the aid of a single relay node as shown in Fig.1. For simplicity, the direct links between transmitters and receivers are ignored as they undergo much larger path attenuation compared with the links via the relay node [12]. The $k$ th transmitter and receiver are equipped with $N_{s k}$ and $N_{d k}$ antennas, respectively, and the number of antennas at the relay node is $N_{r}$.
We assume that the relay node works in the half-duplex mode so the communication between transmitter-receiver pairs is completed in two time slots. In the first time slot, the $k$ th transmitter encodes the $d \times 1$ information-carrying symbol vector $\mathbf{s}_{k}$ with the $N_{s k} \times d$ transmitter precoding matrix $\mathbf{B}_{k}$ before transmitting the $N_{s k} \times 1$ precoded signal vector

$$
\mathbf{x}_{s k}=\mathbf{B}_{k} \mathbf{s}_{k}, \quad k=1, \cdots, K
$$

to the relay node. The received signal vector at the relay node is given by

$$
\mathbf{y}_{r}=\sum_{k=1}^{K} \mathbf{H}_{k} \mathbf{B}_{k} \mathbf{s}_{k}+\mathbf{n}_{r}
$$

where $\mathbf{H}_{k}$ is the $N_{r} \times N_{s k}$ MIMO channel matrix between the $k$ th transmitter and the relay node, $\mathbf{n}_{r}$ is the $N_{r} \times 1$ additive white Gaussian noise (AWGN) vector at the relay node with zero mean and covariance matrix $E\left[\mathbf{n}_{r} \mathbf{n}_{r}^{H}\right]=\sigma_{r}^{2} \mathbf{I}_{N_{r}}$.

In the second time slot, the relay node amplifies the received signal vector with the $N_{r} \times N_{r}$ precoding matrix $\mathbf{F}$ as

$$
\mathbf{x}_{r}=\mathbf{F} \mathbf{y}_{r} .
$$

The precoded signal vector $\mathbf{x}_{r}$ is forwarded to the receivers. The received signal vector at the $k$ th receiver is given by

$$
\mathbf{y}_{d k}=\mathbf{G}_{k} \mathbf{x}_{r}+\mathbf{n}_{d k}, \quad k=1, \cdots, K
$$

where $\mathbf{G}_{k}$ is the $N_{d k} \times N_{r}$ MIMO channel matrix between the relay node and the $k$ th receiver, $\mathbf{n}_{d k}$ is the $N_{d k} \times 1$ AWGN vector at the $k$ th receiver with zero mean and covariance matrix $E\left[\mathbf{n}_{d k} \mathbf{n}_{d k}^{H}\right]=\sigma_{d k}^{2} \mathbf{I}_{N_{d k}}$.

Due to their simplicity, linear receivers are used to retrieve the transmitted signals, and we have $d \leq N_{r}$ and $d \leq N_{d k}$, $k=1, \cdots, K$. The estimated signal vector at the $k$ th receiver can be written as

$$
\hat{\mathbf{s}}_{k}=\mathbf{W}_{k}^{H} \mathbf{y}_{d k}, \quad k=1, \ldots, K
$$

where $\mathbf{W}_{k}$ is the $N_{d k} \times d$ receiver weight matrix. Using (2)(4), the estimated signal vector in (5) becomes

$$
\begin{aligned}
\hat{\mathbf{s}}_{k} & =\underbrace{\mathbf{W}_{k}^{H}\left(\mathbf{G}_{k} \mathbf{F} \sum_{m=1}^{K} \mathbf{H}_{m} \mathbf{B}_{m} \mathbf{s}_{m}+\overline{\mathbf{n}}_{d k}\right)}_{\text {desired signal }} \\
= & \underbrace{\mathbf{W}_{k}^{H} \mathbf{G}_{k} \mathbf{F} \mathbf{H}_{k} \mathbf{B}_{k} \mathbf{s}_{k}}_{\text {interference plus noise }}
\end{aligned}
$$

where $\overline{\mathbf{n}}_{d k} \triangleq \mathbf{G}_{k} \mathbf{F} \mathbf{n}_{r}+\mathbf{n}_{d k}$ is the total noise vector at the $k$ th receiver.

The signal vectors sent by transmitters and the signal vector forwarded from the relay node must satisfy the following transmission power constraints

$$
\begin{aligned}
& \operatorname{tr}\left(\mathbf{B}_{k} E\left[\mathbf{s}_{k} \mathbf{s}_{k}^{H}\right] \mathbf{B}_{k}^{H}\right) \leq P_{s k}, \quad k=1, \cdots, K \\
& \operatorname{tr}\left(\mathbf{F E}\left[\mathbf{y}_{r} \mathbf{y}_{r}^{H}\right] \mathbf{F}^{H}\right) \leq P_{r}
\end{aligned}
$$


where $P_{s k}$ and $P_{r}$ denote the power budget at the $k$ th transmitter and the relay node, respectively, $E\left[\mathbf{s}_{k} \mathbf{s}_{k}^{H}\right]=\mathbf{I}_{d}$ is the covariance matrix of the information-carrying symbol vector at the $k$ th transmitter, and $E\left[\mathbf{y}_{r} \mathbf{y}_{r}^{H}\right]=\sum_{m=1}^{K} \mathbf{H}_{m} \mathbf{B}_{m} \mathbf{B}_{m}^{H} \mathbf{H}_{m}^{H}+$ $\sigma_{r}^{2} \mathbf{I}_{N_{r}}$ is the covariance matrix of the received signal vector at the relay node.

From (6), the MSE of estimating $\mathbf{s}_{k}$ can be calculated as

$$
\begin{aligned}
\operatorname{MSE}_{k}= & \operatorname{tr}\left(E\left[\left(\hat{\mathbf{s}}_{k}-\mathbf{s}_{k}\right)\left(\hat{\mathbf{s}}_{k}-\mathbf{s}_{k}\right)^{H}\right]\right) \\
= & \operatorname{tr}\left(\left(\mathbf{W}_{k}^{H} \mathbf{L}_{k}-\mathbf{I}_{d}\right)\left(\mathbf{W}_{k}^{H} \mathbf{L}_{k}-\mathbf{I}_{d}\right)^{H}\right. \\
& \left.+\mathbf{W}_{k}^{H} \mathbf{C}_{n k} \mathbf{W}_{k}+\mathbf{W}_{k}^{H} \mathbf{\Xi}_{k} \mathbf{W}_{k}\right), k=1, \cdots, K
\end{aligned}
$$

where $\mathbf{L}_{k}$ is the equivalent MIMO channel matrix of the $k$ th transmitter-receiver pair, $\mathbf{C}_{n k}=E\left[\overline{\mathbf{n}}_{d k} \overline{\mathbf{n}}_{d k}^{H}\right]$ is the covariance matrix of the equivalent noise, and $\boldsymbol{\Xi}_{k}$ is the covariance matrix of interference at the $k$ th receiver. They are given respectively as

$$
\begin{aligned}
\mathbf{L}_{k} & =\mathbf{G}_{k} \mathbf{F} \overline{\mathbf{H}}_{k}, \quad k=1, \cdots, K \\
\mathbf{C}_{n k} & =E\left[\left(\mathbf{G}_{k} \mathbf{F} \mathbf{n}_{r}+\mathbf{n}_{d k}\right)\left(\mathbf{G}_{k} \mathbf{F} \mathbf{n}_{r}+\mathbf{n}_{d k}\right)^{H}\right] \\
& =\sigma_{r}^{2} \mathbf{G}_{k} \mathbf{F} \mathbf{F}^{H} \mathbf{G}_{k}^{H}+\sigma_{d k}^{2} \mathbf{I}_{N_{d k}}, \quad k=1, \cdots, K \\
\mathbf{\Xi}_{k} & =\mathbf{G}_{k} \mathbf{F} \sum_{m=1, m \neq k}^{K} \overline{\mathbf{H}}_{m} \overline{\mathbf{H}}_{m}^{H} \mathbf{F}^{H} \mathbf{G}_{k}^{H}, \quad k=1, \cdots, K
\end{aligned}
$$

where $\overline{\mathbf{H}}_{k} \triangleq \mathbf{H}_{k} \mathbf{B}_{k}$ is the equivalent MIMO channel matrix between the $k$ th transmitter and the relay node.

The aim of this paper is to optimize the transmitter precoding matrices $\left\{\mathbf{B}_{k}\right\} \triangleq\left\{\mathbf{B}_{k}, k=1, \cdots, K\right\}$, the relay precoding matrix $\mathbf{F}$, and the receiver weight matrices $\left\{\mathbf{W}_{k}\right\} \triangleq$ $\left\{\mathbf{W}_{k}, k=1, \cdots, K\right\}$, to minimize the sum-MSE of the signal waveform estimation at the receivers under transmission power constraints at the transmitters and the relay node. From (7)(9), the optimal transmitter, relay, and receiver matrices design problem can be written as

$$
\begin{aligned}
\min _{\left\{\mathbf{W}_{k}\right\},\left\{\mathbf{B}_{k}\right\}, \mathbf{F}} & \sum_{k=1}^{K} \mathrm{MSE}_{k} \\
\text { s.t. } & \operatorname{tr}\left(\mathbf{B}_{k} \mathbf{B}_{k}^{H}\right) \leq P_{s k}, k=1, \cdots, K \\
& \operatorname{tr}\left(\mathbf{F} E\left[\mathbf{y}_{r} \mathbf{y}_{r}^{H}\right] \mathbf{F}^{H}\right) \leq P_{r} .
\end{aligned}
$$

\section{Proposed Transmitter, Relay, and Receiver MATRICES Design Algorithms}

The problem (10)-(12) is highly nonconvex with matrix variables, and a globally optimal solution is intractable to obtain. To overcome this challenge, in this section, we propose two iterative algorithms to solve the problem (10)-(12) by optimizing $\left\{\mathbf{W}_{k}\right\},\left\{\mathbf{B}_{k}\right\}$, and $\mathbf{F}$ in an alternating way through solving convex subproblems.

\section{A. Proposed Tri-Step Algorithm}

In each iteration of this algorithm, we first optimize $\left\{\mathbf{W}_{k}\right\}$ based on $\left\{\mathbf{B}_{k}\right\}$ and $\mathbf{F}$ from the previous iteration. Then by using the optimized receiver matrices $\left\{\mathbf{W}_{k}\right\}$ and the transmitter matrices $\left\{\mathbf{B}_{k}\right\}$ from the previous iteration, we optimize the relay matrix $\mathbf{F}$. Finally, we optimize the transmitter matrices $\left\{\mathbf{B}_{k}\right\}$ based on $\left\{\mathbf{W}_{k}\right\}$ and $\mathbf{F}$ obtained from the current iteration.

It can be seen from (7) and (8) that the power constraints are independent of $\left\{\mathbf{W}_{k}\right\}$. Thus, with given relay matrix and transmitter matrices, the optimal linear receiver matrix which minimizes MSE in (9) is the well-known MMSE receiver [16]

$$
\mathbf{W}_{k}=\left(\mathbf{L}_{k} \mathbf{L}_{k}^{H}+\mathbf{C}_{n k}+\mathbf{\Xi}_{k}\right)^{-1} \mathbf{L}_{k}, \quad k=1, \cdots, K .
$$

With given transmitter matrices $\left\{\mathbf{B}_{k}\right\}$ and receiver matrices $\left\{\mathbf{W}_{k}\right\}$ obtained in (13), the sum-MSE SMSE $=\sum_{k=1}^{K} \mathrm{MSE}_{k}$ can be rewritten as a function of $\mathbf{F}$ as

$$
\begin{aligned}
\mathrm{SMSE}= & \sum_{k=1}^{K} \operatorname{tr}\left(\left(\mathbf{W}_{k}^{H} \mathbf{G}_{k} \mathbf{F} \overline{\mathbf{H}}_{k}-\mathbf{I}_{d}\right)\left(\mathbf{W}_{k}^{H} \mathbf{G}_{k} \mathbf{F} \overline{\mathbf{H}}_{k}-\mathbf{I}_{d}\right)^{H}\right. \\
& +\sigma_{r}^{2} \mathbf{W}_{k}^{H} \mathbf{G}_{k} \mathbf{F} \mathbf{F}^{H} \mathbf{G}_{k}^{H} \mathbf{W}_{k}+\sigma_{d k}^{2} \mathbf{W}_{k}^{H} \mathbf{W}_{k} \\
& \left.+\mathbf{W}_{k}^{H} \mathbf{G}_{k} \mathbf{F} \sum_{m=1, m \neq k}^{K} \overline{\mathbf{H}}_{m} \overline{\mathbf{H}}_{m}^{H} \mathbf{F}^{H} \mathbf{G}_{k}^{H} \mathbf{W}_{k}\right)
\end{aligned}
$$

Let us introduce

$$
\begin{aligned}
& \mathbf{H}=\left[\mathbf{H}_{1} \mathbf{B}_{1}, \ldots, \mathbf{H}_{K} \mathbf{B}_{K}\right]=\mathbf{U}_{h} \boldsymbol{\Lambda}_{h} \mathbf{V}_{h}^{H} \\
& \mathbf{G}=\left[\mathbf{G}_{1}^{T}, \ldots, \mathbf{G}_{K}^{T}\right]^{T}=\mathbf{U}_{g} \boldsymbol{\Lambda}_{g} \mathbf{V}_{g}^{H}
\end{aligned}
$$

as the singular-value decomposition (SVD) of the equivalent transmitters-relay channel $\mathbf{H}$ and the equivalent relay-receivers channel G. The dimensions of $\mathbf{U}_{h}, \boldsymbol{\Lambda}_{h}, \mathbf{V}_{h}$ are $N_{r} \times L_{1}$, $L_{1} \times L_{1}, K d \times L_{1}$, respectively and the dimensions of $\mathbf{U}_{g}, \boldsymbol{\Lambda}_{g}$, $\mathbf{V}_{g}$ are $\bar{N}_{d} \times L_{2}, L_{2} \times L_{2}, N_{r} \times L_{2}$, respectively, where $\bar{N}_{d} \triangleq$ $\sum_{k=1}^{K} N_{d k}, L_{1} \triangleq \min \left(K d, N_{r}\right)$, and $L_{2} \triangleq \min \left(\bar{N}_{d}, N_{r}\right)$.

It can be shown similar to [17] that the optimal structure of the relay precoding matrix $\mathbf{F}$ is

$$
\mathbf{F}=\mathbf{V}_{g} \mathbf{A} \mathbf{U}_{h}^{H}
$$

where $\mathbf{A}$ is an $L_{2} \times L_{1}$ matrix. It can be seen from (17) that we only need to optimize $\mathbf{A}$ in order to optimize $\mathbf{F}$. Since the dimension of $\mathbf{A}$ is smaller than or equal to that of $\mathbf{F}$, optimizing A may have a smaller computational complexity than directly optimizing $\mathbf{F}$.

From (15) and (16), we have

$$
\mathbf{H}_{k} \mathbf{B}_{k}=\mathbf{U}_{h} \boldsymbol{\Lambda}_{h} \mathbf{V}_{h, k}^{H}, \mathbf{G}_{k}=\mathbf{U}_{g, k} \boldsymbol{\Lambda}_{g} \mathbf{V}_{g}^{H}, \quad k=1, \cdots, K
$$

where $\mathbf{V}_{h, k}$ contains the $((k-1) d+1)$-th to the $k d$-th rows of $\mathbf{V}_{h}$, and $\mathbf{U}_{g, k}$ contains the $\left(\sum_{i=1}^{k-1} N_{d i}+1\right)$-th to the $\left(\sum_{i=1}^{k} N_{d i}\right)$-th rows of $\mathbf{U}_{g}$, that is, $\mathbf{V}_{h}=\left[\mathbf{V}_{h, 1}^{T}, \ldots, \mathbf{V}_{h, K}^{T}\right]^{T}$, $\mathbf{U}_{g}=\left[\mathbf{U}_{g, 1}^{T}, \ldots, \mathbf{U}_{g, K}^{T}\right]^{T}$. Note that $\mathbf{V}_{h, k}$ and $\mathbf{U}_{g, k}$ have dimensions of $d \times L_{1}$ and $N_{d k} \times L_{2}$, respectively. By substituting (17) into (18), we obtain that for $k=1, \cdots, K$

$$
\begin{aligned}
& \mathbf{G}_{k} \mathbf{F} \overline{\mathbf{H}}_{k}=\mathbf{U}_{g, k} \boldsymbol{\Lambda}_{g} \mathbf{A} \mathbf{\Lambda}_{h} \mathbf{V}_{h, k}^{H} \\
& \mathbf{G}_{k} \mathbf{F} \mathbf{F}^{H} \mathbf{G}_{k}^{H}=\mathbf{U}_{g, k} \boldsymbol{\Lambda}_{g} \mathbf{A} \mathbf{A}^{H} \boldsymbol{\Lambda}_{g} \mathbf{U}_{g, k}^{H} \\
& \mathbf{G}_{k} \mathbf{F} \sum_{m=1, m \neq k}^{K} \overline{\mathbf{H}}_{m} \overline{\mathbf{H}}_{m}^{H} \mathbf{F}^{H} \mathbf{G}_{k}^{H} \\
& =\mathbf{U}_{g, k} \boldsymbol{\Lambda}_{g} \mathbf{A} \sum_{m=1, m \neq k}^{K} \boldsymbol{\Lambda}_{h} \mathbf{V}_{h, m}^{H} \mathbf{V}_{h, m} \boldsymbol{\Lambda}_{h} \mathbf{A}^{H} \boldsymbol{\Lambda}_{g} \mathbf{U}_{g, k}^{H} .
\end{aligned}
$$


Using (19)-(21), the SMSE in (14) becomes

$$
\begin{aligned}
& \mathrm{SMSE}=\sum_{k=1}^{K} \operatorname{tr}\left(\left(\mathbf{W}_{k}^{H} \mathbf{U}_{g, k} \boldsymbol{\Lambda}_{g} \mathbf{A} \boldsymbol{\Lambda}_{h} \mathbf{V}_{h, k}^{H}-\mathbf{I}_{d}\right)\right. \\
& \times\left(\mathbf{W}_{k}^{H} \mathbf{U}_{g, k} \boldsymbol{\Lambda}_{g} \mathbf{A} \boldsymbol{\Lambda}_{h} \mathbf{V}_{h, k}^{H}-\mathbf{I}_{d}\right)^{H} \\
& +\mathbf{W}_{k}^{H} \mathbf{U}_{g, k} \boldsymbol{\Lambda}_{g} \mathbf{A} \sum_{m=1, m \neq k}^{K} \boldsymbol{\Lambda}_{h} \mathbf{V}_{h, m}^{H} \mathbf{V}_{h, m} \boldsymbol{\Lambda}_{h} \mathbf{A}^{H} \boldsymbol{\Lambda}_{g} \mathbf{U}_{g, k}^{H} \mathbf{W}_{k} \\
& \left.+\sigma_{r}^{2} \mathbf{W}_{k}^{H} \mathbf{U}_{g, k} \boldsymbol{\Lambda}_{g} \mathbf{A} \mathbf{A}^{H} \boldsymbol{\Lambda}_{g} \mathbf{U}_{g, k}^{H} \mathbf{W}_{k}+\sigma_{d k}^{2} \mathbf{W}_{k}^{H} \mathbf{W}_{k}\right) .
\end{aligned}
$$

Using the identities of [18]

$$
\begin{aligned}
\operatorname{tr}\left(\mathbf{A}^{T} \mathbf{B}\right) & =(\operatorname{vec}(\mathbf{A}))^{T} \operatorname{vec}(\mathbf{B}) \\
\operatorname{tr}\left(\mathbf{A}^{H} \mathbf{B} \mathbf{A C}\right) & =(\operatorname{vec}(\mathbf{A}))^{H}\left(\mathbf{C}^{T} \otimes \mathbf{B}\right) \operatorname{vec}(\mathbf{A}) \\
\operatorname{vec}(\mathbf{A B C}) & =\left(\mathbf{C}^{T} \otimes \mathbf{A}\right) \operatorname{vec}(\mathbf{B})
\end{aligned}
$$

the SMSE (22) can be represented as a function of $\mathbf{a} \triangleq v e c(\mathbf{A})$ as

$$
\begin{aligned}
\mathrm{SMSE}= & \sum_{k=1}^{K}\left[\left(\mathbf{O}_{k} \mathbf{a}-\operatorname{vec}\left(\mathbf{I}_{d}\right)\right)^{H}\left(\mathbf{O}_{k} \mathbf{a}-\operatorname{vec}\left(\mathbf{I}_{d}\right)\right)\right. \\
& \left.+\mathbf{a}^{H} \mathbf{Q}_{k} \mathbf{a}+\mathbf{a}^{H} \mathbf{S}_{k} \mathbf{a}\right]+t_{1}
\end{aligned}
$$

where $t_{1} \triangleq \sum_{k=1}^{K} \sigma_{d k}^{2} \operatorname{tr}\left(\mathbf{W}_{k}^{H} \mathbf{W}_{k}\right)$ does not depend on $\mathbf{a}$, and for $k=1, \cdots, K$

$$
\begin{aligned}
\mathbf{O}_{k}= & \left(\boldsymbol{\Lambda}_{h} \mathbf{V}_{h, k}^{H}\right)^{T} \otimes\left(\mathbf{W}_{k}^{H} \mathbf{U}_{g, k} \boldsymbol{\Lambda}_{g}\right) \\
\mathbf{Q}_{k}= & \sigma_{r}^{2} \mathbf{I}_{L_{1}} \otimes\left(\boldsymbol{\Lambda}_{g} \mathbf{U}_{g, k}^{H} \mathbf{W}_{k} \mathbf{W}_{k}^{H} \mathbf{U}_{g, k} \boldsymbol{\Lambda}_{g}\right) \\
\mathbf{S}_{k}= & \left(\sum_{m=1, m \neq k}^{K} \boldsymbol{\Lambda}_{h} \mathbf{V}_{h, m}^{H} \mathbf{V}_{h, m} \boldsymbol{\Lambda}_{h}\right)^{T} \\
& \otimes\left(\boldsymbol{\Lambda}_{g} \mathbf{U}_{g, k}^{H} \mathbf{W}_{k} \mathbf{W}_{k}^{H} \mathbf{U}_{g, k} \boldsymbol{\Lambda}_{g}\right) .
\end{aligned}
$$

From (17), the relay node transmission power constraint (8) can be written as

$$
\operatorname{tr}\left(\mathbf{F} E\left[\mathbf{y}_{r} \mathbf{y}_{r}^{H}\right] \mathbf{F}^{H}\right)=\operatorname{tr}\left(\mathbf{A}\left(\boldsymbol{\Lambda}_{h}^{2}+\sigma_{r}^{2} \mathbf{I}_{L_{1}}\right) \mathbf{A}^{H}\right) .
$$

By introducing $\mathbf{D}=\left(\boldsymbol{\Lambda}_{h}^{2}+\sigma_{r}^{2} \mathbf{I}_{L_{1}}\right) \otimes \mathbf{I}_{L_{2}}$, (27) can be rewritten as

$$
\mathbf{a}^{H} \mathbf{D a} \leq P_{r} .
$$

From (26) and (28), the relay matrix optimization problem can be written as

$$
\begin{aligned}
& \underset{\mathbf{a}}{\min } \mathrm{SMSE} \\
& \text { s.t. } \mathbf{a}^{H} \mathbf{D a} \leq P_{r} .
\end{aligned}
$$

The problem (29)-(30) is a quadratically constrained quadratic programming (QCQP) problem [19], which is a convex optimization problem and can be efficiently solved by the interiorpoint method [19]. The problem (29)-(30) can be solved by the CVX MATLAB toolbox for disciplined convex programming [20].
With given receiver matrices $\left\{\mathbf{W}_{k}\right\}$ and the relay matrix $\mathbf{F}$, the sum-MSE can be rewritten as a function of $\left\{\mathbf{B}_{k}\right\}$ as

$$
\begin{aligned}
\mathrm{SMSE}= & \sum_{k=1}^{K} \operatorname{tr}\left(\left(\overline{\mathbf{G}}_{k} \mathbf{F} \mathbf{H}_{k} \mathbf{B}_{k}-\mathbf{I}_{d}\right)\left(\overline{\mathbf{G}}_{k} \mathbf{F} \mathbf{H}_{k} \mathbf{B}_{k}-\mathbf{I}_{d}\right)^{H}\right. \\
& \left.+\overline{\mathbf{G}}_{k} \mathbf{F} \sum_{m=1, m \neq k}^{K} \mathbf{H}_{m} \mathbf{B}_{m} \mathbf{B}_{m}^{H} \mathbf{H}_{m}^{H} \mathbf{F}^{H} \overline{\mathbf{G}}_{k}^{H}\right)+t_{2}
\end{aligned}
$$

where $\overline{\mathbf{G}}_{k}=\mathbf{W}_{k}^{H} \mathbf{G}_{k}$ and $t_{2} \triangleq \sum_{k=1}^{K} \operatorname{tr}\left(\mathbf{W}_{k}^{H} \mathbf{C}_{n k} \mathbf{W}_{k}\right)$ can be ignored in the optimization process as it does not depend on $\left\{\mathbf{B}_{k}\right\}$.

Using the identities in (23)-(25), the SMSE function in (31) can be written as a function of $\mathbf{b}_{k} \triangleq \operatorname{vec}\left(\mathbf{B}_{k}\right)$ as

SMSE

$$
\begin{aligned}
= & \sum_{k=1}^{K}\left[\left(\mathbf{S}_{k} \mathbf{b}_{k}-\operatorname{vec}\left(\mathbf{I}_{d}\right)\right)^{H}\left(\mathbf{S}_{k} \mathbf{b}_{k}-\operatorname{vec}\left(\mathbf{I}_{d}\right)\right)\right. \\
& \left.+\sum_{m=1, m \neq k}^{K} \mathbf{b}_{m}^{H}\left(\mathbf{I}_{d} \otimes \mathbf{H}_{m}^{H} \mathbf{F}^{H} \overline{\mathbf{G}}_{k}^{H} \overline{\mathbf{G}}_{k} \mathbf{F} \mathbf{H}_{m}\right) \mathbf{b}_{m}\right]+t_{2} \\
= & \sum_{k=1}^{K}\left[\left(\mathbf{S}_{k} \mathbf{b}_{k}-\operatorname{vec}\left(\mathbf{I}_{d}\right)\right)^{H}\left(\mathbf{S}_{k} \mathbf{b}_{k}-\operatorname{vec}\left(\mathbf{I}_{d}\right)\right)+\mathbf{b}_{k}^{H} \mathbf{T}_{k} \mathbf{b}_{k}\right]+t_{2}
\end{aligned}
$$

where for $k=1, \cdots, K$

$$
\begin{aligned}
& \mathbf{S}_{k} \triangleq \mathbf{I}_{d} \otimes\left(\overline{\mathbf{G}}_{k} \mathbf{F} \mathbf{H}_{k}\right) \\
& \mathbf{T}_{k} \triangleq \mathbf{I}_{d} \otimes \sum_{m=1, m \neq k}^{K} \mathbf{H}_{k}^{H} \mathbf{F}^{H} \overline{\mathbf{G}}_{m}^{H} \overline{\mathbf{G}}_{m} \mathbf{F} \mathbf{H}_{k} .
\end{aligned}
$$

By introducing $\mathbf{T} \triangleq b d\left(\mathbf{T}_{1}, \cdots, \mathbf{T}_{K}\right)$ and $\overline{\mathbf{S}}_{k} \triangleq$ $\left[\mathbf{S}_{k 1}, \cdots, \mathbf{S}_{k K}\right]$, where $\mathbf{S}_{k k}=\mathbf{S}_{k}$ and $\mathbf{S}_{k i}=\mathbf{0}, i \neq k$, the SMSE function (32) can be written as a function of $\mathbf{b}=\left[\mathbf{b}_{1}^{T}, \mathbf{b}_{2}^{T}, \cdots, \mathbf{b}_{K}^{T}\right]^{T}$ as

$\Phi_{1}(\mathbf{b})=\sum_{k=1}^{K}\left(\overline{\mathbf{S}}_{k} \mathbf{b}-\operatorname{vec}\left(\mathbf{I}_{d}\right)\right)^{H}\left(\overline{\mathbf{S}}_{k} \mathbf{b}-\operatorname{vec}\left(\mathbf{I}_{d}\right)\right)+\mathbf{b}^{H} \mathbf{T} \mathbf{b}$.

Let us introduce $\mathbf{E}_{j}=\mathbf{I}_{d} \otimes\left(\mathbf{H}_{j}^{H} \mathbf{F}^{H} \mathbf{F} \mathbf{H}_{j}\right), \mathbf{E}=$ $b d\left(\mathbf{E}_{1}, \mathbf{E}_{2}, \cdots, \mathbf{E}_{K}\right), \overline{\mathbf{E}}_{i}=b d\left(\overline{\mathbf{E}}_{i 1}, \overline{\mathbf{E}}_{i 2}, \cdots, \overline{\mathbf{E}}_{i K}\right)$, where $\overline{\mathbf{E}}_{i i}=\mathbf{I}_{d N_{s}}$ and $\overline{\mathbf{E}}_{i j}=\mathbf{0}, i \neq j$. The optimal $\mathbf{b}$ can be obtained by solving the following problem

$$
\begin{aligned}
\min _{\mathbf{b}} & \Phi_{1}(\mathbf{b}) \\
\text { s.t. } & \mathbf{b}^{H} \overline{\mathbf{E}}_{k} \mathbf{b} \leq P_{s k}, \quad k=1, \cdots, K \\
& \mathbf{b}^{H} \mathbf{E} \mathbf{b} \leq P_{r}-\sigma_{r}^{2} \operatorname{tr}\left(\mathbf{F F}^{H}\right) .
\end{aligned}
$$

The problem (34)-(36) is a QCQP problem and can be solved by the CVX MATLAB toolbox [20] for disciplined convex programming.

The steps of applying the proposed tri-step algorithm to optimize $\left\{\mathbf{B}_{k}\right\}, \mathbf{F}$, and $\left\{\mathbf{W}_{k}\right\}$ are summarized in Table I, where the superscript $(n)$ denotes the variable at the $n$th iteration, and $\varepsilon$ is a small positive number up to which convergence is acceptable. Since all subproblems (10), (29)(30), and (34)-(36) are convex, the solution to each subproblem is optimal. At the convergence point, since $\left\{\mathbf{W}^{(n)}\right\}, \mathbf{F}^{(n)}$, 
TABLE I

PROCEDURE OF SOLVING THE PROBLEM (10)-(12) BY THE PROPOSED TRI-STEP ALGORITHM.

1) Initialize the algorithm with $\mathbf{F}^{(0)}$ and $\left\{\mathbf{B}_{k}^{(0)}\right\}$ satisfying (7) and (8); Set $n=0$.

2) Obtain $\left\{\mathbf{W}_{k}^{(n+1)}\right\}$ based on (13) with fixed $\mathbf{F}^{(n)}$ and $\left\{\mathbf{B}_{k}^{(n)}\right\}$.

3) Update $\mathbf{A}^{(n+1)}$ through solving the problem (29)-(30) with given $\left\{\mathbf{B}_{k}^{(n)}\right\}$ and $\left\{\mathbf{W}_{k}^{(n+1)}\right\}$.

4) Update $\mathbf{F}^{(n+1)}$ based on (17) from the optimal $\mathbf{A}^{(n+1)}$.

5) Update $\left\{\mathbf{B}_{k}^{(n+1)}\right\}$ by solving the problem (34)-(36) with fixed $\mathbf{F}^{(n+1)}$ and $\left\{\mathbf{W}_{k}^{(n+1)}\right\}$.

6) If $_{\mathrm{MSE}^{(n)}}-\mathrm{MSE}^{(n+1)} \leq \varepsilon$, then end. Otherwise, let $n:=n+1$ and go to Step 2 .

and $\left\{\mathbf{B}_{k}^{(n)}\right\}$ are the optimal solutions to the subproblems (10), (29)-(30), and (34)-(36), respectively, we have

$$
\begin{aligned}
& \operatorname{tr}\left(\nabla_{\mathbf{W}} J\left(\mathbf{\Theta}^{(n)}\right)^{T}\left(\mathbf{W}-\mathbf{W}^{(n)}\right)\right) \geq 0 \\
& \operatorname{tr}\left(\nabla_{\mathbf{F}} J\left(\mathbf{\Theta}^{(n)}\right)^{T}\left(\mathbf{F}-\mathbf{F}^{(n)}\right)\right) \geq 0 \\
& \operatorname{tr}\left(\nabla_{\mathbf{B}} J\left(\mathbf{\Theta}^{(n)}\right)^{T}\left(\mathbf{B}-\mathbf{B}^{(n)}\right)\right) \geq 0
\end{aligned}
$$

where $\mathbf{W} \triangleq\left[\mathbf{W}_{1}, \cdots, \mathbf{W}_{K}\right], \mathbf{W}^{(n)} \triangleq\left[\mathbf{W}_{1}^{(n)}, \cdots, \mathbf{W}_{K}^{(n)}\right]$, $\mathbf{B} \triangleq\left[\mathbf{B}_{1}, \cdots, \mathbf{B}_{K}\right], \mathbf{B}^{(n)} \triangleq\left[\mathbf{B}_{1}^{(n)}, \cdots, \mathbf{B}_{K}^{(n)}\right], \boldsymbol{\Theta}^{(n)} \triangleq$ $\left[\mathbf{W}^{(n)}, \mathbf{B}^{(n)}, \mathbf{F}^{(n)}\right]$, and $\nabla_{\mathbf{X}} J\left(\boldsymbol{\Theta}^{(n)}\right)$ denotes the gradient of the objective function (10) along the direction of $\mathbf{X} \in\left\{\mathbf{W}^{(n)}, \mathbf{B}^{(n)}, \mathbf{F}^{(n)}\right\}$ at $\boldsymbol{\Theta}^{(n)}$. By summing up (37)(39), we have $\operatorname{tr}\left(\nabla J\left(\boldsymbol{\Theta}^{(n)}\right)^{T}\left(\boldsymbol{\Theta}-\boldsymbol{\Theta}^{(n)}\right)\right) \geq 0$, where $\nabla J\left(\boldsymbol{\Theta}^{(n)}\right) \triangleq\left[\nabla_{\mathbf{W}} J\left(\boldsymbol{\Theta}^{(n)}\right), \nabla_{\mathbf{B}} J\left(\boldsymbol{\Theta}^{(n)}\right), \nabla_{\mathbf{F}} J\left(\boldsymbol{\Theta}^{(n)}\right)\right]$, indicating that $\Theta^{(n)}$ is a stationary point of (10) [21].

Now we analyze the computational complexity of the proposed tri-step algorithm assuming $K d \leq N_{r}$ (i.e., $L_{1}=$ $K d$ ) and $\bar{N}_{d} \leq N_{r}$ (i.e., $L_{2}=\bar{N}_{d}$ ). Since the dimension of $\mathbf{b}$ is $\sum_{k=1}^{K} N_{s k} d$ and the dimension of $\mathbf{a}$ is $\bar{N}_{d} K d=\sum_{k=1}^{K} N_{d k} K d$, the computational complexity of solving the QCQP problems (29)-(30) and (34)-(36) using the interior point method [22] is $\mathcal{O}\left(\left(\sum_{k=1}^{K} N_{d k} K d\right)^{3}\right)$ and $\mathcal{O}\left((K+1)^{\frac{1}{2}}\left(\sum_{k=1}^{K} N_{s k} d\right)^{3}\right)$, respectively. Therefore, the computational complexity at each iteration of the proposed tri-step algorithm is $\mathcal{O}\left(\left(\sum_{k=1}^{K} N_{d k} K d\right)^{3}+(K+1)^{\frac{1}{2}}\left(\sum_{k=1}^{K} N_{s k} d\right)^{3}\right)$. It can be seen that the per-iteration computational complexity of the tri-step algorithm can be very high for interference MIMO relay systems with a large number of users $K$, and in this case, the complexity is dominated by the relay matrix optimization.

\section{B. Simplified Relay Matrix Design}

To reduce the computational complexity of optimizing the relay matrix, in this subsection, we develop a simplified relay matrix design algorithm by modifying the power constraint at the relay node, which enables the relay optimization problem to be decomposed into convex subproblems with closed-form solutions.

Substituting the MMSE receiver in (13) to (22) and using
(19)-(21), the SMSE can be rewritten as

$$
\begin{aligned}
& \mathrm{SMSE}=\sum_{k=1}^{K} \operatorname{tr}\left(\mathbf{I}_{d}-\overline{\mathbf{H}}_{k}^{H} \mathbf{F}^{H} \mathbf{G}_{k}^{H}\left(\mathbf{G}_{k} \mathbf{F} \overline{\mathbf{H}}_{k} \overline{\mathbf{H}}_{k}^{H} \mathbf{F}^{H} \mathbf{G}_{k}^{H}\right.\right. \\
& \left.\left.\quad+\mathbf{C}_{n k}+\boldsymbol{\Xi}_{k}\right)^{-1} \mathbf{G}_{k} \mathbf{F} \overline{\mathbf{H}}_{k}\right) \\
& =\sum_{k=1}^{K} \operatorname{tr}\left(\mathbf{I}_{d}-\left(\mathbf{U}_{g, k} \boldsymbol{\Lambda}_{g} \mathbf{A} \boldsymbol{\Lambda}_{h} \mathbf{V}_{h, k}^{H}\right)^{H}\right. \\
& \times\left(\sum_{m=1}^{K} \mathbf{U}_{g, m} \boldsymbol{\Lambda}_{g} \mathbf{A} \boldsymbol{\Lambda}_{h} \mathbf{V}_{h, m}^{H}\left(\mathbf{U}_{g, m} \boldsymbol{\Lambda}_{g} \mathbf{A} \boldsymbol{\Lambda}_{h} \mathbf{V}_{h, m}^{H}\right)^{H}\right. \\
& \left.\left.+\sigma_{r}^{2} \mathbf{U}_{g, k} \boldsymbol{\Lambda}_{g} \mathbf{A} \mathbf{A}^{H} \boldsymbol{\Lambda}_{g} \mathbf{U}_{g, k}^{H}+\sigma_{d k}^{2} \mathbf{I}_{N_{d k}}\right)^{-1} \mathbf{U}_{g, k} \boldsymbol{\Lambda}_{g} \mathbf{A} \boldsymbol{\Lambda}_{h} \mathbf{V}_{h, k}^{H}\right) .
\end{aligned}
$$

Let us introduce

$$
\boldsymbol{\Lambda}_{g} \mathbf{A}=\mathbf{U}_{g}^{H} \mathbf{C}=\sum_{k=1}^{K} \mathbf{U}_{g, k}^{H} \mathbf{C}_{k}
$$

where $\mathbf{C}=\left[\mathbf{C}_{1}^{T}, \mathbf{C}_{2}^{T}, \cdots, \mathbf{C}_{K}^{T}\right]^{T}$ and $\mathbf{C}_{k}$ is an $N_{d k} \times L_{1}$ matrix. Since $\mathbf{U}_{g}^{H} \mathbf{U}_{g}=\mathbf{I}_{L_{2}}$, for any $\mathbf{A}$, we have $\mathbf{C}=$ $\mathbf{U}_{g} \boldsymbol{\Lambda}_{g} \mathbf{A}$. Thus, instead of optimizing $\mathbf{A}$, we can optimize $\left\{\mathbf{C}_{k}\right\} \triangleq\left\{\mathbf{C}_{1}, \cdots, \mathbf{C}_{K}\right\}$.

Using (41), the optimal $\mathbf{F}$ in (17) is

$$
\mathbf{F}=\mathbf{V}_{g} \boldsymbol{\Lambda}_{g}^{-1} \mathbf{U}_{g}^{H} \mathbf{C} \mathbf{U}_{h}^{H} .
$$

By substituting (41) back into (40), we obtained the SMSE as a function of $\left\{\mathbf{C}_{k}\right\}$ as

$$
\mathrm{SMSE}=\sum_{k=1}^{K} \psi_{k}\left(\mathbf{C}_{k}\right)
$$

where

$$
\begin{aligned}
\psi_{k}\left(\mathbf{C}_{k}\right)= & t r\left(\mathbf{I}_{d}-\mathbf{V}_{h, k} \boldsymbol{\Lambda}_{h} \mathbf{C}_{k}^{H}\left(\sum_{m=1}^{K} \mathbf{C}_{k} \boldsymbol{\Lambda}_{h} \mathbf{V}_{h, m}^{H} \mathbf{V}_{h, m} \boldsymbol{\Lambda}_{h} \mathbf{C}_{k}^{H}\right.\right. \\
& \left.\left.+\sigma_{r}^{2} \mathbf{C}_{k} \mathbf{C}_{k}^{H}+\sigma_{d k}^{2} \mathbf{I}_{N_{d k}}\right)^{-1} \mathbf{C}_{k} \boldsymbol{\Lambda}_{h} \mathbf{V}_{h, k}^{H}\right)
\end{aligned}
$$

Interestingly, it can be seen from (43) and (44) that the MSE of the $k$ th transmitter-receiver pair $\psi_{k}$ is a function of $\mathbf{C}_{k}$ only. In other words, the objective function is decomposed in terms of the optimization variable.

From (41), the transmission power constraint at the relay node (27) can be written as

$$
\operatorname{tr}\left(\mathbf{A}\left(\boldsymbol{\Lambda}_{h}^{2}+\sigma_{r}^{2} \mathbf{I}_{L_{1}}\right) \mathbf{A}^{H}\right)=\operatorname{tr}\left(\mathbf{C}^{H} \boldsymbol{\Pi} \mathbf{C} \boldsymbol{\Psi}\right) \leq P_{r}
$$

where $\boldsymbol{\Pi}=\mathbf{U}_{g} \boldsymbol{\Lambda}_{g}^{-2} \mathbf{U}_{g}^{H}$ and $\boldsymbol{\Psi}=\boldsymbol{\Lambda}_{h}^{2}+\sigma_{r}^{2} \mathbf{I}_{L_{1}}$. It can be seen from (45) that $\mathbf{C}_{k}, k=1, \cdots, K$, are coupled through the power constraint. We propose to modify the power constraint (45) by applying the inequality of $\operatorname{tr}(\mathbf{A B}) \leq \operatorname{tr}(\mathbf{A}) \operatorname{tr}(\mathbf{B})$. The transmit power at the relay node becomes

$$
\operatorname{tr}\left(\mathbf{C}^{H} \boldsymbol{\Pi} \mathbf{C} \Psi\right) \leq \operatorname{tr}\left(\mathbf{C} \boldsymbol{\Psi} \mathbf{C}^{H}\right) \operatorname{tr}(\boldsymbol{\Pi}) .
$$

Then the power constraint in (45) is modified to be

$$
\sum_{k=1}^{K} \operatorname{tr}\left(\mathbf{C}_{k} \mathbf{\Psi} \mathbf{C}_{k}^{H}\right) \leq P_{r} / \operatorname{tr}\left(\boldsymbol{\Lambda}_{g}^{-2}\right) .
$$


In fact, (47) imposes a stricter transmission power constraint at the relay node, i.e., if (47) holds, the original power constraint (45) is also satisfied.

Based on (44) and (47), the modified relay matrix optimization problem can be written as

$$
\begin{aligned}
\min _{\left\{\mathbf{C}_{k}\right\}} & \sum_{k=1}^{K} \psi_{k}\left(\mathbf{C}_{k}\right) \\
\text { s.t. } & \sum_{k=1}^{K} \operatorname{tr}\left(\mathbf{C}_{k} \mathbf{\Psi} \mathbf{C}_{k}^{H}\right) \leq \bar{P}_{r}
\end{aligned}
$$

where $\bar{P}_{r}=P_{r} / \operatorname{tr}\left(\boldsymbol{\Lambda}_{g}^{-2}\right)$ is the modified power budget at the relay node. We can see from (48) and (49) that the relay matrix optimization problem can be decomposed into $K$ subproblems where the $k$ th subproblem is to optimize $\mathbf{C}_{k}$ as

$$
\begin{aligned}
& \min _{\mathbf{C}_{k}} \psi_{k}\left(\mathbf{C}_{k}\right) \\
& \text { s.t. } \operatorname{tr}\left(\mathbf{C}_{k} \mathbf{\Psi} \mathbf{C}_{k}^{H}\right) \leq P_{r k} .
\end{aligned}
$$

Here $P_{r k} \geq 0, k=1, \cdots, K$, and $\sum_{k=1}^{K} P_{r k}=\bar{P}_{r}$. Interestingly, in the following, we show that the problem (50)-(51) can be viewed as the MMSE-based relay matrix optimization problem for a single-user two-hop MIMO relay system, which is convex and has a closed-form solution.

Let us introduce the following matrices for $k=1, \cdots, K$

$$
\begin{aligned}
\mathbf{J}_{r k} & =\sum_{m=1, m \neq k}^{K} \boldsymbol{\Lambda}_{h} \mathbf{V}_{h, m}^{H} \mathbf{V}_{h, m} \boldsymbol{\Lambda}_{h}+\sigma_{r}^{2} \mathbf{I}_{L_{1}} \\
\mathbf{X}_{k} & =\mathbf{J}_{r k}^{-\frac{1}{2}} \boldsymbol{\Lambda}_{h} \mathbf{V}_{h, k}^{H} \\
\mathbf{Y}_{k} & =\mathbf{C}_{k} \mathbf{J}_{r k}^{\frac{1}{2}}
\end{aligned}
$$

where the dimensions of $\mathbf{X}_{k}$ and $\mathbf{Y}_{k}$ are $L_{1} \times d$ and $N_{d k} \times L_{1}$, respectively. The MSE for the $k$ th transmitter-receiver pair becomes

$$
\begin{aligned}
f_{k}\left(\mathbf{Y}_{k}\right)= & \operatorname{tr}\left(\mathbf{I}_{d}-\mathbf{X}_{k}^{H} \mathbf{Y}_{k}^{H}\left(\mathbf{Y}_{k} \mathbf{X}_{k} \mathbf{X}_{k}^{H} \mathbf{Y}_{k}^{H}\right.\right. \\
& \left.\left.+\mathbf{Y}_{k} \mathbf{Y}_{k}^{H}+\sigma_{d k}^{2} \mathbf{I}_{N_{d k}}\right)^{-1} \mathbf{Y}_{k} \mathbf{X}_{k}\right) \\
= & \operatorname{tr}\left(\left(\mathbf{I}_{d}+\mathbf{X}_{k}^{H} \mathbf{Y}_{k}^{H}\left(\mathbf{Y}_{k} \mathbf{Y}_{k}^{H}+\sigma_{d k}^{2} \mathbf{I}_{N_{d k}}\right)^{-1} \mathbf{Y}_{k} \mathbf{X}_{k}\right)^{-1}\right)(55)
\end{aligned}
$$

and the power constraint (51) becomes

$$
\operatorname{tr}\left(\mathbf{Y}_{k}\left(\mathbf{X}_{k} \mathbf{X}_{k}^{H}+\mathbf{I}_{L_{1}}\right) \mathbf{Y}_{k}^{H}\right) \leq P_{r k} .
$$

Using (55) and (56), the problem (50)-(51) can be equivalently rewritten as

$$
\begin{aligned}
& \min _{\mathbf{Y}_{k}} f_{k}\left(\mathbf{Y}_{k}\right) \\
& \text { s.t. } \operatorname{tr}\left(\mathbf{Y}_{k}\left(\mathbf{X}_{k} \mathbf{X}_{k}^{H}+\mathbf{I}_{L_{1}}\right) \mathbf{Y}_{k}^{H}\right) \leq P_{r k} .
\end{aligned}
$$

The problem (57)-(58) is the MMSE-based relay matrix optimization problem for a single-user two-hop MIMO relay system [5], [6] with the first hop channel $\mathbf{X}_{k}$, the relay matrix $\mathbf{Y}_{k}$ and the second hop channel $\mathbf{I}_{N_{d k}}$. It can be shown similar to [5], [6] that the optimal structure of $\mathbf{Y}_{k}$ is

$$
\mathbf{Y}_{k}=\left[\mathbf{I}_{d}, \quad \mathbf{0}_{d \times\left(N_{d k}-d\right)}\right]^{T} \boldsymbol{\Lambda}_{y, k} \mathbf{U}_{x, k}^{H}
$$

where $\mathbf{X}_{k}=\mathbf{U}_{x, k} \boldsymbol{\Lambda}_{x, k} \mathbf{V}_{x, k}^{H}$ is the SVD of $\mathbf{X}_{k}$, and the dimensions of $\mathbf{U}_{x, k}, \boldsymbol{\Lambda}_{x, k}$ and $\mathbf{V}_{x, k}$ are $L_{1} \times d, d \times d$, and $d \times d$, respectively.

By substituting (59) back into (48) and (49), the relay matrix optimization problem becomes

$$
\begin{aligned}
\min _{\left\{\boldsymbol{\Lambda}_{y, k}\right\}} & \sum_{k=1}^{K} \operatorname{tr}\left(\left(\mathbf{I}_{d}+\boldsymbol{\Lambda}_{x, k}^{2}\left(\mathbf{I}_{d}+\sigma_{d k}^{2} \boldsymbol{\Lambda}_{y, k}^{-2}\right)^{-1}\right)^{-1}\right) \\
\text { s.t. } & \sum_{k=1}^{K} \operatorname{tr}\left(\boldsymbol{\Lambda}_{y, k}^{2}\left(\boldsymbol{\Lambda}_{x, k}^{2}+\mathbf{I}_{d}\right)\right) \leq \bar{P}_{r}
\end{aligned}
$$

where $\left\{\boldsymbol{\Lambda}_{y, k}\right\} \triangleq\left\{\boldsymbol{\Lambda}_{y, 1}, \cdots, \boldsymbol{\Lambda}_{y, K}\right\}$. The problem (60)-(61) can be equivalently rewritten as the following problem with scalar variables

$$
\begin{aligned}
\min _{\left\{\lambda_{y, k, i}\right\}} & \sum_{k=1}^{K} \sum_{i=1}^{d}\left(1+\frac{\lambda_{x, k, i}^{2} \lambda_{y, k, i}^{2}}{\lambda_{y, k, i}^{2}+\sigma_{d k}^{2}}\right)^{-1} \\
\text { s.t. } & \sum_{k=1}^{K} \sum_{i=1}^{d} \lambda_{y, k, i}^{2}\left(\lambda_{x, k, i}^{2}+1\right) \leq \bar{P}_{r} \\
& \lambda_{y, k, i} \geq 0, k=1, \cdots, K, i=1, \cdots, d
\end{aligned}
$$

where $\lambda_{x, k, i}$ and $\lambda_{y, k, i}, i=1, \cdots, d$, are the $i$ th diagonal element of $\boldsymbol{\Lambda}_{x, k}$ and $\boldsymbol{\Lambda}_{y, k}$, respectively, and $\left\{\lambda_{y, k, i}\right\} \triangleq$ $\left\{\lambda_{y, 1,1}, \cdots, \lambda_{y, K, d}\right\}$.

The problem (62)-(64) has the well-known water-filling solution and is given by

$$
\begin{gathered}
\lambda_{y, k, i}=\sqrt{\frac{1}{\lambda_{x, k, i}^{2}+1}\left[\sqrt{\frac{\sigma_{d k}^{2} \lambda_{x, k, i}^{2}}{\left(\lambda_{x, k, i}^{2}+1\right) \beta}}-\sigma_{d k}^{2}\right]^{\dagger}} \\
k=1, \cdots, K, i=1, \cdots, d
\end{gathered}
$$

where $[x]^{\dagger} \triangleq \max (x, 0)$, and $\beta>0$ is the solution to the following equation

$$
\sum_{k=1}^{K} \sum_{i=1}^{d}\left[\sqrt{\frac{\sigma_{d k}^{2} \lambda_{x, k, i}^{2}}{\left(\lambda_{x, k, i}^{2}+1\right) \beta}}-\sigma_{d k}^{2}\right]^{\dagger}=\bar{P}_{r} .
$$

As the left-hand side of (66) is a non-increasing function of $\beta$, it can be efficiently solved by the bisection method [19]. Finally, the relay precoding matrix can be obtaining from (42), (54), (59), and (65).

We would like to note that the idea of the simplified relay matrix design is to convert the complicated relay matrix optimization problem for interference MIMO relay system to the much simpler relay matrix design for single user MIMO relay system, through modifying the power constraint. As the problem (57)-(58) is the MMSE-based relay matrix optimization problem for a single-user two-hop MIMO relay system, the derivations (60)-(66) are similar to that in [6].

The transmitter matrices $\left\{\mathbf{B}_{k}\right\}$ and receiver matrices $\left\{\mathbf{W}_{k}\right\}$ can be optimized through (34)-(36) and (13), respectively. The steps of applying the simplified relay matrix design to solve the transceiver optimization problem are summarized in Table II. Since the dimension of $\left\{\lambda_{y, k, i}\right\}$ is $K d$, the computational complexity of solving the problem (62)-(64) is $\mathcal{O}(K d)$. When $L_{1}=K d$ (as in the complexity analysis in Section III-A), the SVD of $\mathbf{X}_{k}$ has a complexity order of $\mathcal{O}\left(K d^{3}\right)$. Therefore, the 
TABLE II

PROCEDURE OF SOLVING THE PROBLEM (10)-(12) THROUGH THE SIMPLIFIED RELAY MATRIX DESIGN.

1) Initialize the algorithm with $\mathbf{F}^{(0)}$ and $\left\{\mathbf{B}_{k}^{(0)}\right\}$ satisfying (7) and (8); Set $n=0$.

2) Obtain $\left\{\mathbf{W}_{k}^{(n+1)}\right\}$ based on (13) with fixed $\mathbf{F}^{(n)}$ and $\left\{\mathbf{B}_{k}^{(n)}\right\}$.

3) Solve the problems (62)-(64) with given $\left\{\mathbf{B}_{k}^{(n)}\right\}$ to find $\left\{\lambda_{y, k, i}\right\}$ and update $\mathbf{F}^{(n+1)}$ through (42), (54), (59), and (65).

4) Update $\left\{\mathbf{B}_{k}^{(n+1)}\right\}$ by solving the problem (34)-(36) with fixed $\mathbf{F}^{(n+1)}$ and $\left\{\mathbf{W}_{k}^{(n+1)}\right\}$.

5) $\operatorname{If~}_{\mathrm{MSE}^{(n)}}-\mathrm{MSE}^{(n+1)} \leq \varepsilon$, then end.

Otherwise, let $n:=n+1$ and go to Step 2 .

complexity of the simplified relay matrix design is $\mathcal{O}\left(K^{2} d^{3}\right)$, which is much lower than the computational complexity of the relay matrix design in the previous subsection. However, we will see through numerical simulations that the proposed algorithm in Table I has a better MSE and BER performance than the algorithm in Table II. Such performance-complexity tradeoff is very useful for practical interference MIMO relay communication systems.

\section{Extensions of the Proposed Algorithms}

In this section, we show that both proposed algorithms can be extended to interference MIMO relay communication systems with imperfect CSI. Moreover, the proposed tristep algorithm can be extended to interference MIMO relay systems with multiple relay nodes.

\section{A. Interference MIMO relay systems with CSI mismatch}

In the case of CSI mismatch, the true channel matrices can be modelled as the well-known Gaussian-Kronecker model [14]

$$
\begin{aligned}
& \mathbf{H}_{k} \sim \mathcal{C N}\left(\hat{\mathbf{H}}_{k}, \boldsymbol{\Theta}_{h, k} \otimes \boldsymbol{\Phi}_{h, k}\right), k=1, \cdots, K \\
& \mathbf{G}_{k} \sim \mathcal{C N}\left(\hat{\mathbf{G}}_{k}, \boldsymbol{\Theta}_{g, k} \otimes \boldsymbol{\Phi}_{g, k}\right), k=1, \cdots, K
\end{aligned}
$$

where $\hat{\mathbf{H}}_{k}$ and $\hat{\mathbf{G}}_{k}$ are the estimated channel matrices, $\boldsymbol{\Theta}_{h, k}$ and $\boldsymbol{\Phi}_{h, k}$ denote the covariance matrix of channel estimation error seen from transmitter side and receiver side, respectively. From (67)-(68), we have

$$
\begin{aligned}
\mathbf{H}_{k} & =\hat{\mathbf{H}}_{k}+\mathbf{A}_{\boldsymbol{\Phi}_{h, k}} \mathbf{H}_{w, k} \mathbf{A}_{\boldsymbol{\Theta}_{h, k}}^{H}, k=1, \cdots, K \\
\mathbf{G}_{k} & =\hat{\mathbf{G}}_{k}+\mathbf{A}_{\boldsymbol{\Phi}_{g, k}} \mathbf{G}_{w, k} \mathbf{A}_{\boldsymbol{\Theta}_{g, k}}^{H}, k=1, \cdots, K
\end{aligned}
$$

where $\mathbf{A}_{\boldsymbol{\Phi}_{i, k}} \mathbf{A}_{\boldsymbol{\Phi}_{i, k}}^{H}=\boldsymbol{\Phi}_{i, k}, \mathbf{A}_{\boldsymbol{\Theta}_{i, k}} \mathbf{A}_{\Theta_{i, k}}^{H}=\boldsymbol{\Theta}_{i, k}^{T}, i=h, g$, $\mathbf{H}_{w, k}$ and $\mathbf{G}_{w, k}$ are Gaussian random matrices with i.i.d. zero mean and unit variance entries and are the unknown parts in the CSI mismatch. The dimensions of $\boldsymbol{\Theta}_{h, k}$ is $N_{s k} \times N_{s k}$, $\boldsymbol{\Theta}_{g, k}$ and $\boldsymbol{\Phi}_{h, k}$ have a dimension of $N_{r} \times N_{r}$, while $\boldsymbol{\Phi}_{g, k}$ is an $N_{d k} \times N_{d k}$ matrix. As the exact CSI is unknown, in the following, we show that both proposed algorithms can be extended to design statistically robust transceivers.

It is shown in [23] that for a random matrix $\mathbf{H} \sim$ $\mathcal{C N}(\hat{\mathbf{H}}, \boldsymbol{\Theta} \otimes \boldsymbol{\Phi})$, there is

$$
E_{H}\left[\mathbf{H X} \mathbf{H}^{H}\right]=\hat{\mathbf{H}} \mathbf{X} \hat{\mathbf{H}}^{H}+\operatorname{tr}\left(\mathbf{X} \Theta^{T}\right) \boldsymbol{\Phi}
$$

where $E_{H}[\cdot]$ stands for the expectation with respect to the random matrix $\mathbf{H}$. Considering the CSI mismatch (67), (68) and using (69), we have for $m, k=1, \cdots, K$

$$
\begin{aligned}
& E_{G, H}\left[\mathbf{L}_{k}\right]=\hat{\mathbf{G}}_{k} \mathbf{F} \hat{\mathbf{H}}_{k} \mathbf{B}_{k} \triangleq \hat{\mathbf{L}}_{k} \\
& E_{G, H}\left[\mathbf{G}_{k} \mathbf{F} \overline{\mathbf{H}}_{m} \overline{\mathbf{H}}_{m}^{H} \mathbf{F}^{H} \mathbf{G}_{k}^{H}\right] \\
& =E_{G}\left[\mathbf{G}_{k} \mathbf{F}\left(\hat{\mathbf{H}}_{m} \mathbf{B}_{m} \mathbf{B}_{m}^{H} \hat{\mathbf{H}}_{m}^{H}+\alpha_{m} \boldsymbol{\Phi}_{h, m}\right) \mathbf{F}^{H} \mathbf{G}_{k}^{H}\right] \\
& =\hat{\mathbf{G}}_{k} \mathbf{F}\left(\hat{\mathbf{H}}_{m} \mathbf{B}_{m} \mathbf{B}_{m}^{H} \hat{\mathbf{H}}_{m}^{H}+\alpha_{m} \boldsymbol{\Phi}_{h, m}\right) \mathbf{F}^{H} \hat{\mathbf{G}}_{k}^{H}+\beta_{m, k} \mathbf{\Phi}_{g, k} \\
& \sigma_{r}^{2} E_{G}\left[\mathbf{G}_{k} \mathbf{F} \mathbf{F}^{H} \mathbf{G}_{k}^{H}\right]=\sigma_{r}^{2} \hat{\mathbf{G}}_{k} \mathbf{F} \mathbf{F}^{H} \hat{\mathbf{G}}_{k}^{H}+\gamma_{k} \boldsymbol{\Phi}_{g, k}
\end{aligned}
$$

where for $m, k=1, \cdots, K$

$$
\begin{aligned}
\alpha_{m} & =\operatorname{tr}\left(\mathbf{B}_{m} \mathbf{B}_{m}^{H} \boldsymbol{\Theta}_{h, m}^{T}\right) \\
\beta_{m, k} & =\operatorname{tr}\left(\mathbf{F}\left(\hat{\mathbf{H}}_{m} \mathbf{B}_{m} \mathbf{B}_{m}^{H} \hat{\mathbf{H}}_{m}^{H}+\alpha_{m} \boldsymbol{\Phi}_{h, m}\right) \mathbf{F}^{H} \boldsymbol{\Theta}_{g, k}^{T}\right) \\
\gamma_{k} & =\sigma_{r}^{2} \operatorname{tr}\left(\mathbf{F} \mathbf{F}^{H} \boldsymbol{\Theta}_{g, k}^{T}\right) .
\end{aligned}
$$

Using (70)-(72), the statistical expectation of the sum-MSE in (14) with respect to $\mathbf{H}_{k}$ and $\mathbf{G}_{k}, k=1, \cdots, K$, can be calculated as

$$
\begin{aligned}
& E_{G, H}[\mathrm{SMSE}] \\
= & \sum_{k=1}^{K} \operatorname{tr}\left(\left(\mathbf{W}_{k}^{H} \hat{\mathbf{L}}_{k}-\mathbf{I}_{d}\right)\left(\mathbf{W}_{k}^{H} \hat{\mathbf{L}}_{k}-\mathbf{I}_{d}\right)^{H}+\mathbf{W}_{k}^{H}\left(\hat{\mathbf{C}}_{n, k}+\hat{\mathbf{\Xi}}_{k}\right.\right. \\
+ & \left.\left.\gamma_{k} \boldsymbol{\Phi}_{g, k}+\sum_{m=1}^{K}\left(\alpha_{m} \hat{\mathbf{G}}_{k} \mathbf{F} \boldsymbol{\Phi}_{h, m} \mathbf{F}^{H} \hat{\mathbf{G}}_{k}^{H}+\beta_{m, k} \boldsymbol{\Phi}_{g, k}\right)\right) \mathbf{W}_{k}\right)
\end{aligned}
$$

where for $k=1, \cdots, K$

$$
\begin{aligned}
\hat{\mathbf{C}}_{n, k} & =\sigma_{r}^{2} \hat{\mathbf{G}}_{k} \mathbf{F} \mathbf{F}^{H} \hat{\mathbf{G}}_{k}^{H}+\sigma_{d k}^{2} \mathbf{I}_{N_{d k}} \\
\hat{\boldsymbol{\Xi}}_{k} & =\hat{\mathbf{G}}_{k} \mathbf{F} \sum_{m=1, m \neq k}^{K} \hat{\mathbf{H}}_{m} \mathbf{B}_{m} \mathbf{B}_{m}^{H} \hat{\mathbf{H}}_{m}^{H} \mathbf{F}^{H} \hat{\mathbf{G}}_{k}^{H} .
\end{aligned}
$$

By introducing

$$
\begin{aligned}
& \mathbf{P}_{1} \triangleq \sum_{m=1}^{K} \alpha_{m} \boldsymbol{\Phi}_{h, m}+\sigma_{r}^{2} \mathbf{I}_{N_{r}} \\
& \mathbf{P}_{2, k} \triangleq\left(\sum_{m=1}^{K} \beta_{m, k}+\gamma_{k}\right) \boldsymbol{\Phi}_{g, k}+\sigma_{d k}^{2} \mathbf{I}_{N_{d k}}, \quad k=1, \cdots, K
\end{aligned}
$$

we can rewrite (73) as

$$
\begin{array}{r}
E_{G, H}[\mathrm{SMSE}]=\sum_{k=1}^{K} \operatorname{tr}\left(\left(\mathbf{W}_{k}^{H} \hat{\mathbf{L}}_{k}-\mathbf{I}_{d}\right)\left(\mathbf{W}_{k}^{H} \hat{\mathbf{L}}_{k}-\mathbf{I}_{d}\right)^{H}\right. \\
\left.+\mathbf{W}_{k}^{H}\left(\hat{\mathbf{G}}_{k} \mathbf{F} \mathbf{P}_{1} \mathbf{F}^{H} \hat{\mathbf{G}}_{k}^{H}+\hat{\mathbf{\Xi}}_{k}+\mathbf{P}_{2, k}\right) \mathbf{W}_{k}\right) \cdot(74)
\end{array}
$$

Let us introduce $\tilde{\mathbf{F}} \triangleq \mathbf{F} \mathbf{P}_{1}^{\frac{1}{2}}$ and for $k=1, \cdots, K$

$$
\tilde{\mathbf{W}}_{k}^{H} \triangleq \mathbf{W}_{k}^{H} \mathbf{P}_{2, k}^{\frac{1}{2}}, \quad \tilde{\mathbf{H}}_{k} \triangleq \mathbf{P}_{1}^{-\frac{1}{2}} \hat{\mathbf{H}}_{k}, \quad \tilde{\mathbf{G}}_{k} \triangleq \mathbf{P}_{2, k}^{-\frac{1}{2}} \hat{\mathbf{G}}_{k} .
$$

We can rewrite (74) as

$$
\begin{aligned}
E_{G, H}[\mathrm{SMSE}]= & \sum_{k=1}^{K} \operatorname{tr}\left(\left(\tilde{\mathbf{W}}_{k}^{H} \tilde{\mathbf{L}}_{k}-\mathbf{I}_{d}\right)\left(\tilde{\mathbf{W}}_{k}^{H} \tilde{\mathbf{L}}_{k}-\mathbf{I}_{d}\right)^{H}\right. \\
& \left.+\tilde{\mathbf{W}}_{k}^{H}\left(\tilde{\mathbf{C}}_{n, k}+\tilde{\boldsymbol{\Xi}}_{k}\right) \tilde{\mathbf{W}}_{k}\right)
\end{aligned}
$$


where for $k=1, \cdots, K$

$$
\begin{aligned}
\tilde{\mathbf{L}}_{k} & =\tilde{\mathbf{G}}_{k} \tilde{\mathbf{F}} \tilde{\mathbf{H}}_{k} \mathbf{B}_{k} \\
\tilde{\mathbf{C}}_{n, k} & =\tilde{\mathbf{G}}_{k} \tilde{\mathbf{F}} \tilde{\mathbf{F}}^{H} \tilde{\mathbf{G}}_{k}^{H}+\mathbf{I}_{N_{d k}} \\
\tilde{\mathbf{\Xi}}_{k} & =\tilde{\mathbf{G}}_{k} \tilde{\mathbf{F}} \sum_{m=1, m \neq k}^{K} \tilde{\mathbf{H}}_{m} \mathbf{B}_{m} \mathbf{B}_{m}^{H} \tilde{\mathbf{H}}_{m} \tilde{\mathbf{F}}^{H} \tilde{\mathbf{G}}_{k}^{H} .
\end{aligned}
$$

In the case of CSI mismatch, the power constraint in (12) becomes

$$
\begin{aligned}
& \operatorname{tr}\left(\mathbf{F} E_{H}\left[\mathbf{y}_{r} \mathbf{y}_{r}^{H}\right] \mathbf{F}^{H}\right) \\
= & \operatorname{tr}\left(\mathbf{F}\left(\sum_{m=1}^{K}\left(\hat{\mathbf{H}}_{m} \mathbf{B}_{m} \mathbf{B}_{m}^{H} \hat{\mathbf{H}}_{m}^{H}+\alpha_{m} \boldsymbol{\Phi}_{h, m}\right)+\sigma_{r}^{2} \mathbf{I}_{N_{r}}\right) \mathbf{F}^{H}\right) \\
= & \operatorname{tr}\left(\tilde{\mathbf{F}}\left(\sum_{m=1}^{K} \tilde{\mathbf{H}}_{m} \mathbf{B}_{m} \mathbf{B}_{m}^{H} \tilde{\mathbf{H}}_{m}^{H}+\mathbf{I}_{N_{r}}\right) \tilde{\mathbf{F}}^{H}\right) .
\end{aligned}
$$

Using (75) and (76), the statistically robust transmitter, relay, and receiver matrices design problem for interference MIMO relay systems under CSI mismatch can be equivalently written as

$$
\begin{aligned}
\min _{\left\{\tilde{\mathbf{W}}_{k}\right\},\left\{\mathbf{B}_{k}\right\}, \tilde{\mathbf{F}}} & E_{G, H}[\mathrm{SMSE}] \\
\text { s.t. } & \operatorname{tr}\left(\mathbf{B}_{k} \mathbf{B}_{k}^{H}\right) \leq P_{s k}, k=1, \cdots, K \\
& \operatorname{tr}\left(\tilde{\mathbf{F}}\left(\sum_{m=1}^{K} \tilde{\mathbf{H}}_{m} \mathbf{B}_{m} \mathbf{B}_{m}^{H} \tilde{\mathbf{H}}_{m}^{H}+\mathbf{I}_{N_{r}}\right) \tilde{\mathbf{F}}^{H}\right) \leq P_{r}
\end{aligned}
$$

where $\left\{\tilde{\mathbf{W}}_{k}\right\} \triangleq\left\{\tilde{\mathbf{W}}_{1}, \cdots, \tilde{\mathbf{W}}_{K}\right\}$. By comparing the problem (77)-(79) with the problem (10)-(12), it can be seen that the problem (77)-(79) is in fact a transmitter optimization problem for an "equivalent" interference MIMO relay system where the transmitter-relay and relay-receiver channels are $\tilde{\mathbf{H}}_{k}$ and $\tilde{\mathbf{G}}_{k}, k=1, \cdots, K$, respectively, the relay precoding matrix is $\tilde{\mathbf{F}}$, and the transmitter and receiver matrices are $\mathbf{B}_{k}$ and $\tilde{\mathbf{W}}_{k}, k=1, \cdots, K$, respectively. Therefore, both proposed algorithms can be applied to solve the problem (77)-(79).

\section{B. Interference MIMO relay systems with multiple relay nodes}

The proposed tri-step algorithm can be easily extended to interference MIMO relay systems with multiple relay nodes. Let us consider a system with $L$ relay nodes, where $\mathbf{F}_{l}$ denotes the precoding matrix at the $l$ th relay node, $\mathbf{H}_{l k}$ and $\mathbf{G}_{k l}$ are the channel matrices from the $k$ th transmitter to the $l$ th relay, and from the $l$ th relay to the $k$ th receiver, respectively. Let us introduce the following SVDs for $l=1, \cdots, L$

$$
\begin{aligned}
& {\left[\mathbf{H}_{l 1} \mathbf{B}_{1}, \ldots, \mathbf{H}_{l K} \mathbf{B}_{K}\right]=\mathbf{U}_{h, l} \boldsymbol{\Lambda}_{h, l} \mathbf{V}_{h, l}^{H}} \\
& {\left[\mathbf{G}_{1 l}^{T}, \ldots, \mathbf{G}_{l K}^{T}\right]^{T}=\mathbf{U}_{g, l} \boldsymbol{\Lambda}_{g, l} \mathbf{V}_{g, l}^{H} .}
\end{aligned}
$$

Similar to (17), it can be shown that the optimal structure of $\mathbf{F}_{l}$ is

$$
\mathbf{F}_{l}=\mathbf{V}_{g, l} \mathbf{A}_{l} \mathbf{U}_{h, l}^{H}, \quad l=1, \cdots, L .
$$

Similar to the procedure in Table I, in each iteration of the tristep algorithm, we first update $\left\{\mathbf{W}_{k}\right\}$ with given $\left\{\mathbf{B}_{k}\right\}$ and $\left\{\mathbf{F}_{l}\right\} \triangleq\left\{\mathbf{F}_{1}, \cdots, \mathbf{F}_{L}\right\}$. Then we update each relay matrix $\mathbf{F}_{l}$ based on its optimal structure (80) with fixed $\left\{\mathbf{W}_{k}\right\},\left\{\mathbf{B}_{k}\right\}$, and other relay matrices $\mathbf{F}_{m}, m=1, \cdots, L, m \neq l$. Finally, we optimize $\left\{\mathbf{B}_{k}\right\}$ with given $\left\{\mathbf{W}_{k}\right\}$ and $\left\{\mathbf{F}_{l}\right\}$.

On the other hand, the proposed simplified relay matrix design cannot be straightforwardly extended to multi-relay systems. Similar to (42), the optimal $\mathbf{F}_{l}$ in (80) can be written as

$$
\mathbf{F}_{l}=\mathbf{V}_{g, l} \Lambda_{g, l}^{-1} \mathbf{U}_{g, l}^{H} \mathbf{C}_{l} \mathbf{U}_{h, l}^{H}, \quad l=1, \cdots, L
$$

where $\mathbf{C}_{l}=\left[\mathbf{C}_{l, 1}^{T}, \cdots, \mathbf{C}_{l, K}^{T}\right]^{T}$. It can be shown that the MSE of the $k$ th transmitter-receiver pair is a function of $\mathbf{C}_{1, k}, \mathbf{C}_{2, k}, \cdots, \mathbf{C}_{L, k}$. However, the power constraint at the $l$ th relay node is a function of $\mathbf{C}_{l, 1}, \mathbf{C}_{l, 2}, \cdots, \mathbf{C}_{l, K}$. Thus, unlike a single-relay system, the optimal relay matrix design problem in multi-relay systems cannot be easily decomposed into $K$ subproblems with closed-form solutions, due to the couplings among all $\mathbf{C}_{l, k}$. Developing a simplified relay matrices design algorithm for an interference MIMO relay system with multiple relay nodes is an interesting future research topic.

\section{NUMERICAL EXAMPLES}

In this section, we study the performance of the proposed joint transceiver matrices design algorithms for interference MIMO relay systems in Table I (Algorithm 1) and Table II (Algorithm 2) through numerical simulations. We consider an interference MIMO relay system with $d=3$, where all transmitters and receivers have the same number of antennas, i.e., $N_{s k}=N_{d k}=4, k=1, \cdots, K$. Unless explicitly mentioned, the relay node has $N_{r}=20$ antennas. We also assume that all transmitters have the same power budget of $P_{s k}=20 \mathrm{~dB}$, $k=1, \cdots, K$. All channel matrices have i.i.d. complex Gaussian entries with zero mean and unit variance, and all noises are i.i.d. Gaussian with zero mean and unit variance. The QPSK constellations are used to modulate the source symbols. All simulation results are averaged over $5 \times 10^{5}$ independent channel realizations. Both proposed algorithms are initialized with $\mathbf{F}^{(0)}=\sqrt{P_{r} / \operatorname{tr}\left(\sum_{k=1}^{K} P_{s k} \mathbf{H}_{k} \mathbf{H}_{k}^{H} / N_{s k}+\mathbf{I}_{N_{r}}\right)} \mathbf{I}_{N_{r}}$ and $\mathbf{B}_{k}^{(0)}=\sqrt{P_{s k} / N_{s k}} \mathbf{I}_{N_{s k}}, k=1, \cdots, K$. As a benchmark, the performance of the proposed algorithms is compared with the joint power control and transceiver-relay beamforming (TxRxBF) algorithm developed in [12] and the total leakage minimization (TLM) algorithm developed in [13].

In the first example, we study the convergence speed of the proposed algorithms. Fig. 2 and Fig. 3 show respectively the normalized SMSE and BER performance of the two proposed algorithms versus the number of iterations at various levels of $P_{r}$ with $K=2$. It can be seen that at all $P_{r}$ levels, the proposed Algorithm 2 converges within two iterations. Interestingly, the convergence speed of the proposed Algorithm 1 decreases with increasing $P_{r}$. However, even at $P_{r}=20 \mathrm{~dB}$, the proposed Algorithm 1 converges around 10 iterations. In fact, the decreasing of the MSE and BER are negligible after seven iterations. Thus, we suggest that only seven iterations are needed for the proposed Algorithm 1 to achieve a good performance.

In the second example, we compare the performance of two proposed algorithms with the TLM algorithm. Fig. 4 shows the 


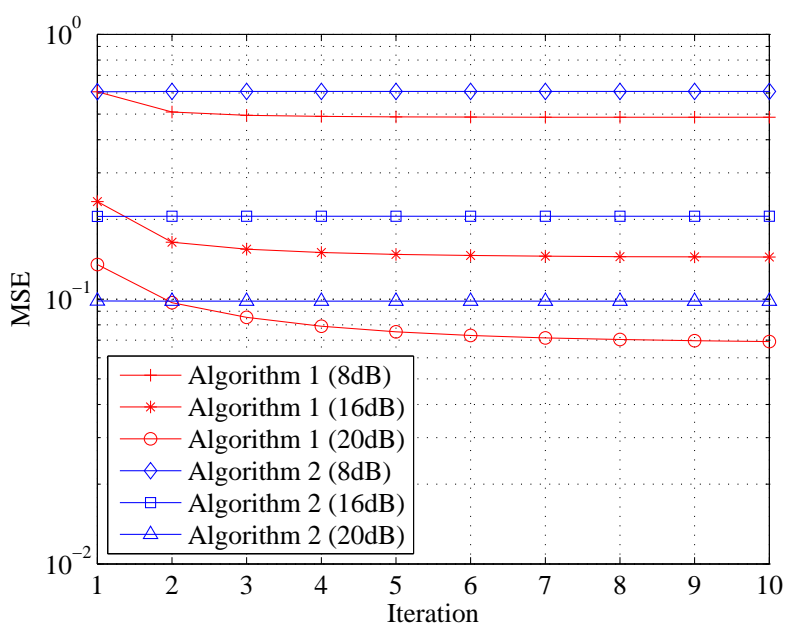

Fig. 2. Example 1: MSE versus the number of iterations, $K=2$.

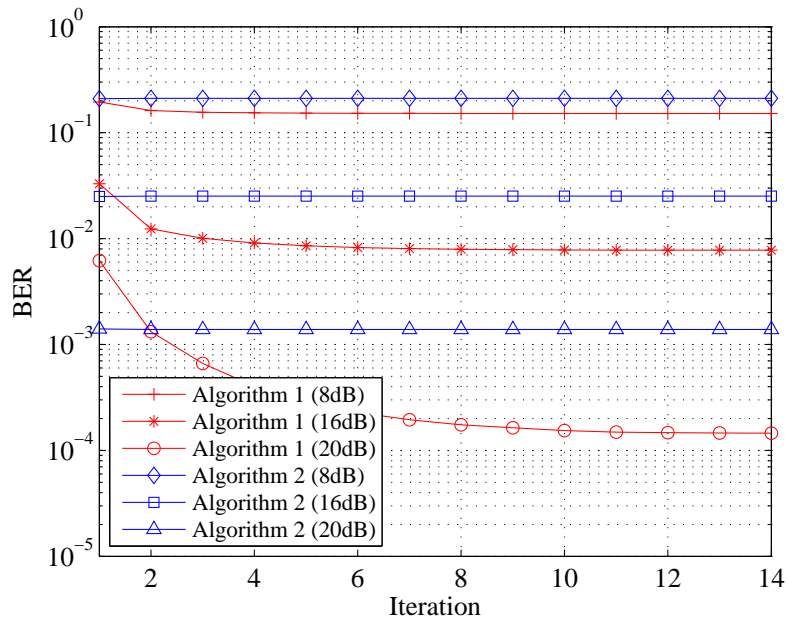

Fig. 3. Example 1: BER versus the number of iterations, $K=2$.

normalized SMSE performance of the three algorithms tested versus $P_{r}$ with $K=2$. It can be seen that both proposed algorithms outperform the TLM algorithm throughout the whole $P_{r}$ range when $N_{r}=20$. While the proposed Algorithm 1 has a better MSE performance than the proposed Algorithm 2 at convergence, the latter algorithm has a lower computational complexity.

For this example, the BER of all transmitter-receiver pairs versus $P_{r}$ yielded by the three algorithms is shown in Fig. 5. It can be seen that both proposed algorithms yield smaller BER than the TLM algorithm over the whole $P_{r}$ range when $N_{r}=20$. Moreover, when it converges, the proposed Algorithm 1 has a better BER performance than the proposed Algorithm 2 at a higher computational complexity. We also observe from Fig. 5 that both transmitter-receiver pairs achieve almost identical BER, indicating that both proposed algorithms are fair to all links.

It can be seen from Figs. 4 and 5 that the MSE and BER of both proposed algorithms are higher at $N_{r}=5$ compared with those at $N_{r}=20$. This is because as the number of

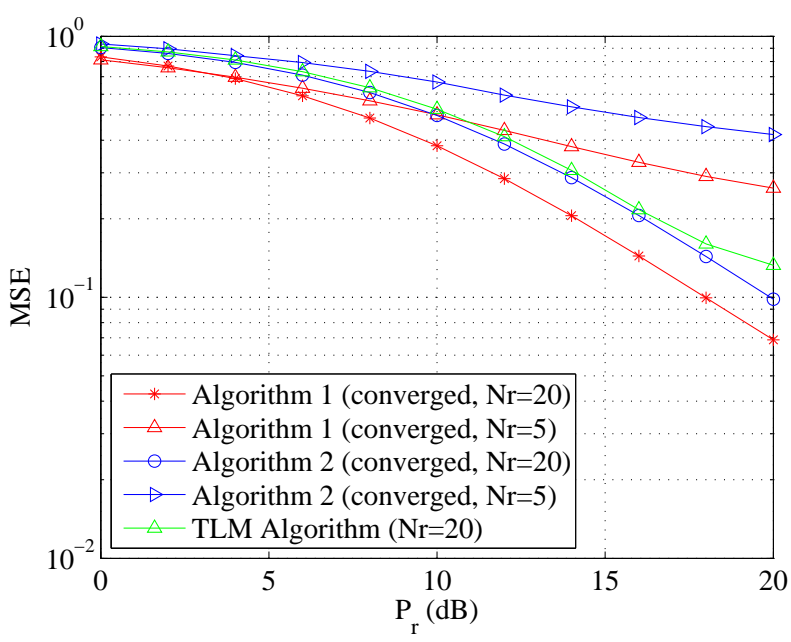

Fig. 4. Example 2: Comparison of MSE versus $P_{r}, K=2$.

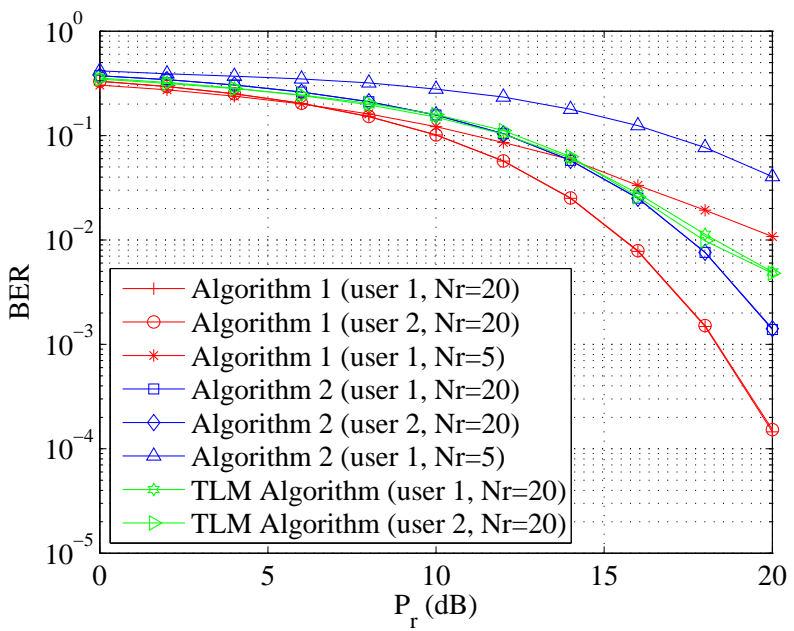

Fig. 5. Example 2: BER versus $P_{r}$ for each transmitter-receiver pair, $K=2$.

independent data streams from all transmitters is $K d=6$, there is no degree-of-freedom at the relay node to separate data streams when $N_{r}=5$.

In the third example, we study the performance of the proposed algorithms with different number of transmitterreceiver pairs $K$. The normalized SMSE performance of both proposed algorithms versus $P_{r}$ is shown in Fig. 6 for $K=2,3,4$. As expected, for both algorithms, the MSE increases with $K$. Moreover, the proposed Algorithm 1 has better MSE performance than the proposed Algorithm 2 for all $K$ values.

In the fourth example, we study the effect of CSI mismatch on the performance of two proposed algorithms. We assume that the channel estimation error at the transmitter side is uncorrelated, i.e., $\boldsymbol{\Theta}_{h, k}=\sigma_{e}^{2} \mathbf{I}_{N_{s k}}$ and $\boldsymbol{\Theta}_{g, k}=\sigma_{e}^{2} \mathbf{I}_{N_{r}}$ where $\sigma_{e}^{2}$ measures the variance of the channel estimation error. The covariance matrices of the channel estimation error at the 


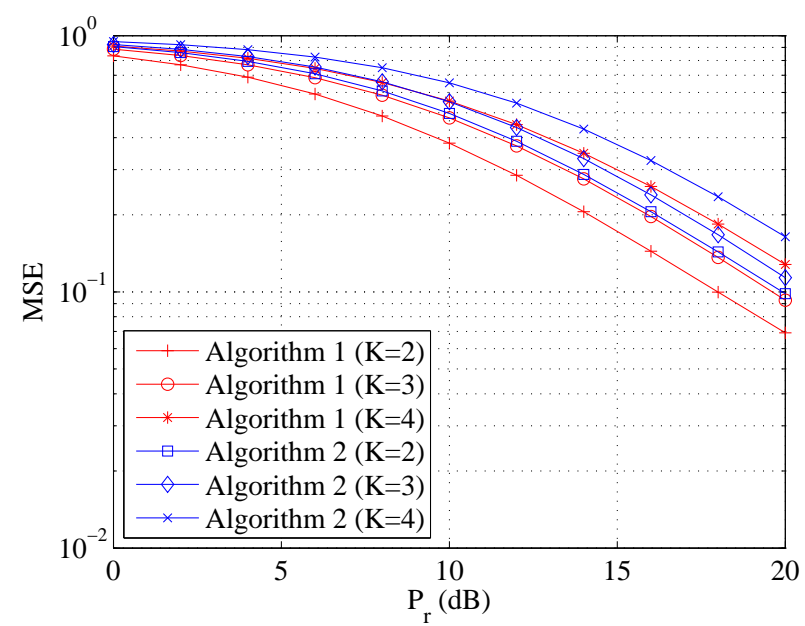

Fig. 6. Example 3: MSE versus $P_{r}$ for different $K$.

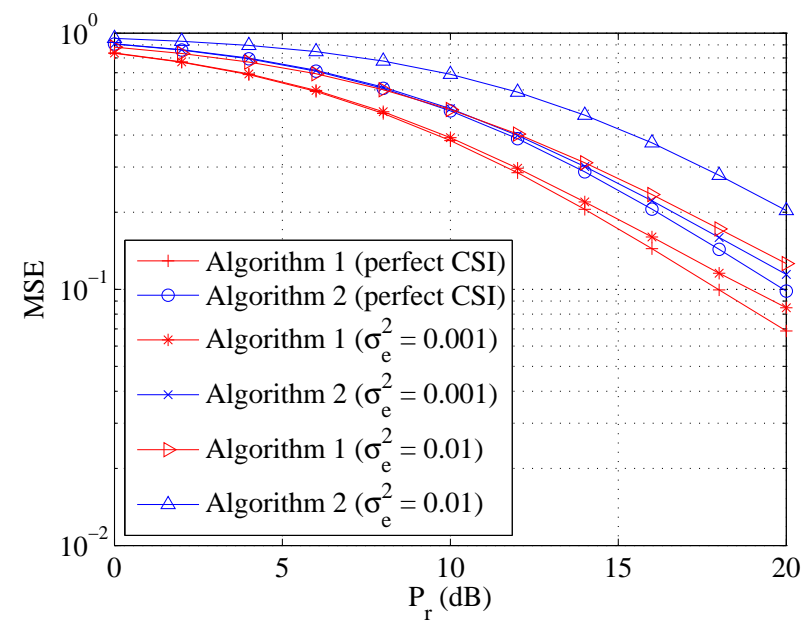

Fig. 7. Example 4: Effect of CSI mismatch on the proposed algorithms.

receiver side is set as

$$
\boldsymbol{\Phi}_{h, k}=\left[\begin{array}{cccc}
1 & \phi_{h} & \phi_{h}^{2} & \phi_{h}^{3} \\
\phi_{h} & 1 & \phi_{h} & \phi_{h}^{2} \\
\phi_{h}^{2} & \phi_{h} & 1 & \phi_{h} \\
\phi_{h}^{3} & \phi_{h}^{2} & \phi_{h} & 1
\end{array}\right], \quad \boldsymbol{\Phi}_{g, k}=\left[\begin{array}{cccc}
1 & \phi_{g} & \phi_{g}^{2} & \phi_{g}^{3} \\
\phi_{g} & 1 & \phi_{g} & \phi_{g}^{2} \\
\phi_{g}^{2} & \phi_{g} & 1 & \phi_{g} \\
\phi_{g}^{3} & \phi_{g}^{2} & \phi_{g} & 1
\end{array}\right]
$$

where we choose $\phi_{h}=\phi_{g}=0.45$ in the simulation. Fig. 7 shows the performance of the two proposed algorithms under $\sigma_{e}^{2}=0.01$ and 0.001. As expected, it can be seen from Fig. 7 that the MSE increases with $\sigma_{e}^{2}$. When $\sigma_{e}^{2}$ is small, the system MSE is very close to that of the system with the perfect CSI, indicating that both proposed algorithms are efficient in the case of CSI mismatch.

In the last example, we compare the BER performance of the proposed algorithms with the TLM algorithm and the TxRxBF algorithm in [12]. As the TxRxBF algorithm is only applicable to an interference MIMO relay system with $d=1$, thus in this example, we choose $d=1$. It can be seen from Fig. 8 that both proposed algorithms and the TxRxBF algorithm yield smaller system BER than the TLM algorithm. Moreover, the proposed Algorithm 1 outperforms the TxRxBF

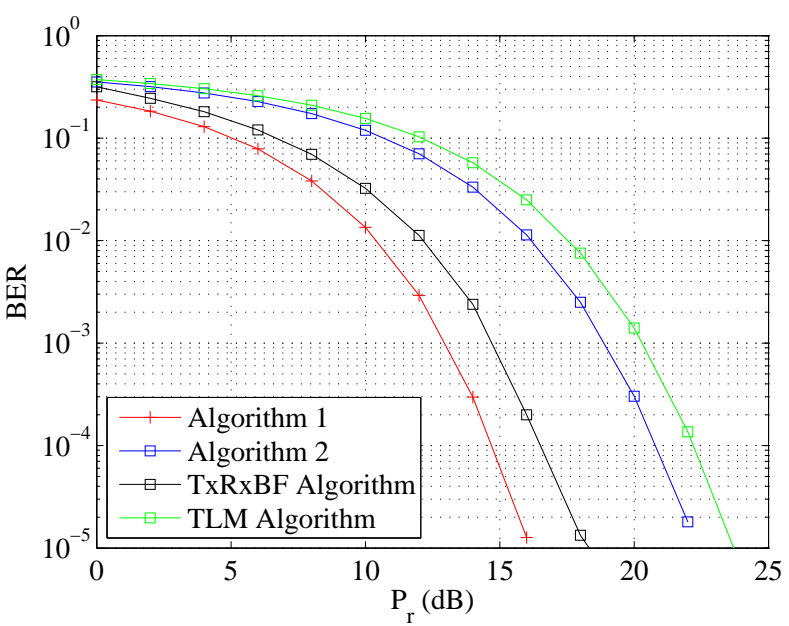

Fig. 8. Example 5: Comparison of BER versus $P_{r}, K=2$.

algorithm, while the TxRxBF algorithm has a better BER performance than the proposed Algorithm 2. Note that the computational complexity of the TxRxBF algorithm is much higher than that of the proposed Algorithm 2.

\section{CONCLUSION}

In this paper, we have presented two algorithms for jointly optimizing the transmitter, relay, and receiver matrices of interference MIMO relay systems. In particular, the optimal structure of the relay precoding matrix has been derived to reduce the computational complexity. Moreover, by modifying the power constraint at the relay node, a simplified relay matrix design has been proposed which has a closed-form solution. Numerical simulation results show that the proposed algorithms converge quickly after a few iterations. The proposed Algorithm 1 has a better MSE and BER performance than the proposed Algorithm 2 at a higher computational complexity.

\section{REFERENCES}

[1] D. P. Palomar, J. M. Cioffi, and M. A. Lagunas, "Joint Tx-Rx beamforming design for multicarrier MIMO channels: A unified framework for convex optimization," IEEE Trans. Signal Process., vol. 51, pp. 23812401, Sep. 2003.

[2] Y. Fan and J. Thompson, "MIMO configurations for relay channels: Theory and practice," IEEE Trans. Wireless Commun., vol. 6, pp. 17741786, May 2007.

[3] L. Sanguinetti, A. A. D'Amico, and Y. Rong, "A tutorial on the optimization of amplify-and-forward MIMO relay systems," IEEE $J$. Selet. Areas Commun., vol. 30, pp. 1331-1346, Sep. 2012.

[4] G. Kramer, M. Gastpar, and P. Gupta, "Cooperative strategies and capacity theorems for relay networks," IEEE Trans. Inf. Theory, vol. 51, pp. 3037-3063, Sep. 2005.

[5] W. Guan and H. Luo, "Joint MMSE transceiver design in nonregenerative MIMO relay systems," IEEE Commun. Lett., vol. 12, pp. 517-519, Jul. 2008.

[6] Y. Rong, X. Tang, and Y. Hua, "A unified framework for optimizing linear nonregenerative multicarrier MIMO relay communication systems," IEEE Trans. Signal Process., vol. 57, pp. 4837-4851, Dec. 2009.

[7] C. Zhao and B. Champagne, "Joint design of multiple non-regenerative MIMO relaying matrices with power constraints," IEEE Trans. Signal Process., vol. 61, pp. 4861-4873, Oct. 2013.

[8] A. Toding, M. R. A. Khandaker, and Y. Rong, "Joint source and relay optimization for parallel MIMO relay networks," EURASIP Journal Advances Signal Process., 2012:174. 
[9] M. Maddah-Ali, A. Motahari, and A. Khandani, "Communication over MIMO X channels: Interference alignment, decomposition, and performance analysis," IEEE Trans. Inf. Theory, vol. 54, pp. 3457-3470, Aug. 2008.

[10] B. Nourani, S. Motahari, and A. Khandani, "Relay-aided interference alignment for the quasi-static X channel," in Proc. IEEE Int. Symposium Inf. Theory, Seoul, Korea, Jun. 28-Jul. 3, 2009, pp. 1764-1768.

[11] X. Wang, Y.-P. Zhang, P. Zhang, and X. Ren, "Relay-aided interference alignment for MIMO cellular networks," in Proc. IEEE Int. Symposium Inf. Theory, Cambridge, MA, Jul. 1-6, 2012, pp. 2641-2645.

[12] M. Khandaker and Y. Rong, "Interference MIMO relay channel: Joint power control and transceiver-relay beamforming," IEEE Trans. Signal Process. vol. 60, pp. 6509-6518, Dec. 2012.

[13] K. T. Truong, P. Sartori, and R. W. Heath, "Cooperative algorithms for MIMO amplify-and-forward relay networks," IEEE Trans. Signal Process. vol. 61, pp. 1272-1287, Mar. 2013.

[14] J. Yang and B. Champagne, "Joint transceiver optimization for MIMO multiuser relaying networks with channel uncertainties," in Proc. IEEE Vehicular Technology Conference (VTC Fall), Vancouver, BC, Canada, Sep. 14-17, 2014, pp. 1-6.

[15] C. Xing, S. Li, Z. Fei, and J. Kuang, "How to understand linear minimum mean-square-error transceiver design for multiple-input-multiple-output systems from quadratic matrix programming," IET Communications, vol. 7, no. 12, pp. 1231-1242, 2013.

[16] S. M. Kay, Fundamentals of Statistical Signal Processing: Estimation Theory. Englewood Cilffs, NJ: Prentice Hall, 1993.

[17] Y. Rong, "Linear non-regenerative multicarrier MIMO relay communications based on MMSE criterion," IEEE Trans. Commun., vol. 58, pp. 1918-1923, Jul. 2010.

[18] J. W. Brewer, "Kronecker products and matrix calculus in system theory," IEEE Trans. Circuits Syst., vol. 25, pp. 772-781, Sep. 1978.

[19] S. Boyd and L. Vandenberghe, Convex Optimization. Cambridge, U. K.: Cambridge University Press, 2004.

[20] M. Grant and S. Boyd, "Cvx: Matlab software for disciplined convex programming (webpage and software)," [Online]. Available: http://cvxr.com/cvx, Apr. 2010.

[21] R. Wang and M. Tao, "Joint source and relay precoding designs for MIMO two-way relaying based on MSE criterion," IEEE Trans. Signal Process. vol. 60, pp. 1352-1365, Mar. 2012.

[22] Y. Nesterov and A. Nemirovski, Interior Point Polynomial Algorithms in Convex Programming. Philadelphia, PA: SIAM, 1994.

[23] A. Gupta and D. Nagar, Matrix Variate Distributions. London, U. K.: Chapman \& Hall/CRC, 2000.

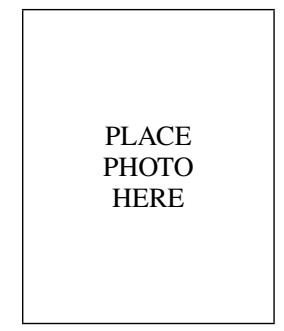

Khoa Xuan Nguyen received the B.E. (Hons.) degree in electronic and communication engineering from Curtin University, Australia, in 2011. He is currently working toward the Ph.D. degree at the Department of Electrical and Computer Engineering, Curtin University, Australia. His research interests include transceiver optimisation for interference MIMO relay communication systems.

Mr. Nguyen was awarded the Curtin International Postgraduate Research Scholarship (CIPRS) for his Ph.D. study in 2011.

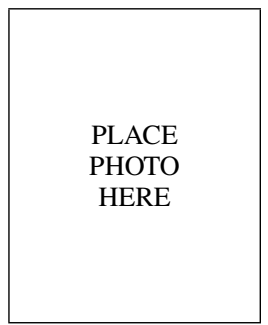

Yue Rong (S'03-M'06-SM'11) received the Ph.D. degree (summa cum laude) in electrical engineering from the Darmstadt University of Technology, Darmstadt, Germany, in 2005.

He was a Post-Doctoral Researcher with the Department of Electrical Engineering, University of California, Riverside, from February 2006 to November 2007. Since December 2007, he has been with the Department of Electrical and Computer Engineering, Curtin University, Bentley, Australia, where he is currently an Associate Professor. His research interests include signal processing for communications, wireless communications, underwater acoustic communications, applications of linear algebra and optimization methods, and statistical and array signal processing. He has published over 120 journal and conference paper in these areas.

Dr. Rong was a recipient of the Best Paper Award at the 2011 International Conference on Wireless Communications and Signal Processing, the Best Paper Award at the 2010 Asia-Pacific Conference on Communications, and the Young Researcher of the Year Award of the Faculty of Science and Engineering at Curtin University in 2010. He is an Associate Editor of the IEEE Transactions on Signal Processing. He was an Editor of the IEEE WIRELESS COMMUNICATIONS LETTERS from 2012 to 2014, a Guest Editor of the IEEE Journal on SELECTED AREAS IN COMMUNICATIONS special issue on theories and methods for advanced wireless relays, and was a TPC Member for the IEEE ICC, WCSP, IWCMC, and ChinaCom.

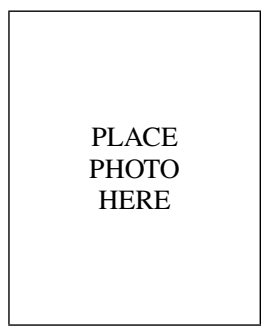

Sven Nordholm (M'90-SM'05) received his Ph.D. in signal processing in 1992 Licentiate of engineering in 1989 and MscEE (Civilingenjr) in 1983 all from Lund University, Sweden. He is Professor at Curtin University, Perth, Australia.

$\mathrm{He}$ was one of the founding members of the Department of Signal Processing, Blekinge Institute of Technology, BTH, Sweden in 1990. At BTH he held positions as Lecturer, Senior Lecturer, Associate Professor and Professor. Since 1999, he has been at Curtin University of Technology in Perth, Western Australia. From 1999-2002, he was director of ATRI and Professor at Curtin University of Technology. From 2002-2009, he was director Signal Processing Laboratory, WATRI, Western Australian Telecommunication Research Institute, a joint institute between The University of Western Australia and Curtin University. From 2009, he has been a Professor in Electrical and Computer Engineering in Curtin University. During 2012-2013, he served as Head of Department. He has also been Chief Scientist and co-founder of a start-up company Sensear, which provides voice communication in extreme noise conditions. He is also founder and director of a hearing aid company Hearmore. He is an associate editor EURASIP Journal on Advances in Signal Processing and Journal of Franklin Institute. He is a Senior Member of IEEE and a member of IEEE TC AASP.

His main research efforts have been spent in the fields of speech enhancement, adaptive and optimum microphone arrays, acoustic echo cancellation, adaptive signal processing, sub-band adaptive filtering and filter design. 